\title{
On Fuzzy Critical Path Method Based On Ranking Of Various Type-2 Fuzzy Quantities Using Centroid Of Centroids
}

\author{
A. Hari Ganesh, A. Helen Shobana
}

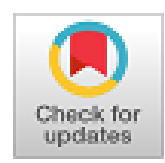

\begin{abstract}
This paper proposes a simple approach to critical path analysis in a project network with activity times being intervals and which are converted into various Type-2 fuzzy quantities. The idea is based on generalized type-2 trapezoidal, hexagonal and octagonal fuzzy numbers and its ranking. The explicit form of membership functions of the type-2 fuzzy activity times is not required in the proposed approach. Moreover, the method is very simple and the numerical example is given for demonstrating and comparing the proposed approach with generalized type-2 trapezoidal, hexagonal and octagonal fuzzy numbers through proposed ranking function.
\end{abstract}

Keywords : Fuzzy Critical Path, Generalized Type-2 Hexagonal Fuzzy Number, Generalized Type-2 Octagonal Fuzzy Number, Generalized Type-2 Trapezoidal Fuzzy Number, Ranking Functionss.

\section{INTRODUCTION}

Critical path method (CPM) is an algorithm based project scheduling method designed to analyze and represent the tasks involved in completing a given complicated projects including aerospace, defense, construction and software development etc. The main objective of this method is to assess the performance of the project and to identify the important activities on the critical path so that the resources could be utilized on these activities in order to optimize the parameters involved in the project. In the literature, stochastic approaches such as Program Evaluation and Review Technique (PERT) and Monte Carlo simulation based probability theories in certain environment have been widely used in many of the research articles so far. In the recent years, fuzzy variables have been using for modeling the duration of the activities in the project network instead of stochastic variables under uncertainty. Fuzzy critical path method was introduced since the late 1970s and several approaches are proposed over the past years. In Fuzzy Critical Path Problems, path lengths are considered as fuzzy numbers. The decision makers assess the path lengths with fuzzy numbers and the path selection will eventually lead to

Revised Manuscript Received on October 30, 2019.

* Correspondence Author

A. Hari Ganesh*, P.G. \& Research Department of Mathematics, Poombuhar College (Autonomous), Melaiyur, Nagapattinam, Tamil Nadu, India.

A. Helen Shobana, P.G. \& Research Department of Mathematics, Bon Secours College for Women, Thanjavur, Tamil Nadu, India.

(C) The Authors. Published by Blue Eyes Intelligence Engineering and Sciences Publication (BEIESP). This is an open access article under the CC BY-NC-ND license (http://creativecommons.org/licenses/by-nc-nd/4.0/) the ranking of corresponding fuzzy numbers. Thus, specific ranking of fuzzy numbers is an important process in identifying critical path under fuzzy environment. So Fuzzy Critical Path Problems are solved using Ranking Methods. From the beginning itself, many authors have been involving in solving critical path problems based on various ranking methods. Among the existing methods, some approaches are surveyed and listed as follows:

In 2010, Sireesha and Ravi Shankar [12] developed a new method based on fuzzy theory to solve the project scheduling problem without computing forward and backward pass calculations under uncertainty. Shakeela Sathish and Ganesan [11] proposed a new approach to critical path analysis in project network based on fuzzy ranking method to determine the fuzzy critical path of project network without converting the fuzzy activity times to classical numbers in 2011. In 2013, Ravi Shankar [10] developed a new fuzzy critical path method by applying a proposed approach of ranking of fuzzy numbers using centroid of centroids of fuzzy number to its distance from original point and Elizabeth and Sujatha [3] proposed a new ranking method based on acceptability and decision maker risk index to identify the fuzzy critical path in which they presented the fuzzy critical length in the nature of fuzzy membership function in the same year. In 2014, Jayagowri [5] made a novel approach to find the critical path based on intuitionistic trapezoidal fuzzy number in a directed acyclic graph. Subsequently, Oladeinde [8] carried out fuzzy critical path analysis of a project network based on metric distance ranking technique for ordering fuzzy numbers during the forward and backward pass computations to obtain the earliest start, earliest finish, latest start and latest finish time of the project's activities. However, Stephen Dinagar and Abirami [13-15] proposed two different approaches such as ranking value of a fuzzy number approach and a distinct approach to critical path analysis in project networks in 2014. Moreover, in this work activity times are uncertain and are represented by interval valued fuzzy numbers. Latterly, they have studied fuzzy critical path in project network based on new ranking value of LR type interval valued fuzzy numbers with the help of proposed arithmetic operations in 2016. A comparison also has been made between Sanguansat and Chen ranking method with their proposed ranking approach in this work. In their recent study, a one more new method has been made to find the critical path and the total float for project scheduling problems with the aid of interval valued fuzzy numbers based on new distance based ranking function. On the basis of method of magnitude for ranking of fuzzy numbers,

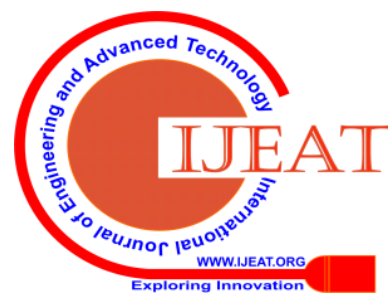


[16] this paper has dealt a novel method for solving critical path problems with fuzzy duration by Vimala and Krishna Prabha in 2015. In 2016, Narayanamoorthy and Maheswari [7] proposed a modified ranking approach to determine the critical path in fuzzy project network where the duration of each activity time is represented by an octagonal fuzzy number. Next, Pushparaj and John Pal [9] presented an algorithm to perform critical path method based on metric distance ranking of fuzzy numbers in fuzzy environment. We [4] also proposed an algorithm to find the shortest path of a networking problem based on interval numbers using novel ranking function of generalized type -2 hexagonal fuzzy numbers in the same year. Moreover, the arithmetic operations of generalized type -2 hexagonal fuzzy numbers also presented in this paper for the calculation of critical path. Recently in 2017, Anusuya and Balasowandari [1] proposed a new approach to find critical path using various distances of trapezoidal type-2 fuzzy numbers.

In this paper, we present generalized type-2 fuzzy arithmetic operations and their application to a critical path problem based on fuzzy float time. In particular, the paper proposes an algorithm for finding the fuzzy critical path based on interval numbers and various type 2 fuzzy quantities using centroid based ranking method. To illustrate the proposed method, a numerical example is presented and solved as a type- 2 critical path problem. Moreover, the generalized type-2 trapezoidal, hexagonal and octagonal fuzzy numbers are compared through this example based on proposed ranking function.

\section{PRELIMINARIES}

\section{A. Fuzzy Set[6]}

A fuzzy set $A$ on the universe of discourse $\mathrm{X}$ can be defined by its membership function $\mu_{\tilde{A}}(x)$, and characterized as

$$
A=\left\{\left(x, \mu_{\AA}(x)\right) \mid \forall x \in X_{*} \mu_{\AA}(x) \in[0,1]\right\}
$$

\section{B. Type-2 Fuzzy set[6]}

A type-2 fuzzy set $A$ on the universe of discourse $\mathrm{X}$ can be defined by its membership function $\mu_{\widetilde{\tilde{A}}}(x, u)$, and characterized as follows:

$$
A=\left\{\left((x, u), \mu_{A}(x, u)\right) \mid \forall x \in X_{v} \forall u \in J_{x} \subseteq[0,1]\right\}
$$

where $0 \leq \mu_{\widetilde{\widetilde{A}}}(x, u) \leq 1$, the subinterval $J_{x}$ is called the primary membership of $x$, and $\mu_{A}\left(x_{x} u\right)$ is called the secondary membership function.

\section{Type-2 fuzzy number[17]}

A type-2 fuzzy set $A$ is called a type-2 fuzzy number if the following conditions are satisfied

(i) $A$ is normal

(ii) $A$ is convex

(iii) The support of $A$ is closed and bounded

\section{Generalized type-2 trapezoidal fuzzy number[2]}

A Generalized Type-2 Trapezoidal fuzzy number $A$ is defined by
$A=\left\{\left(x, \mu_{A}^{L}(x), \mu_{A}^{M}(x), \mu_{A}^{N}(x), \mu_{A}^{P}(x)\right)\right\}$ and $\mu_{A}^{L}(x) \leq \mu_{A}^{M}(x) \leq \mu_{A}^{N}(x) \leq \mu_{A}^{p}(x)$ for all $x \in \mathrm{R}$.

It is denoted by $A=\left(\left(a_{1}^{L}, a_{2}^{L}, a_{\mathrm{a}}^{L}, a_{4}^{L} ; \lambda_{A}\right),\left(a_{1}^{M}, a_{2}^{M}, a_{2}^{M}, a_{4}^{M} ; \sigma_{A}\right)\right.$, $\left.\left(a_{1}^{N}, a_{2}^{N}, a_{2}^{N}, a_{4}^{N} ; \tau_{A}\right),\left(a_{1}^{P}, a_{2}^{p}, a_{2}^{p}, a_{4}^{p} ; p_{A}\right) ; w_{A}\right)$

\section{E. Generalized type-2 hexagonal fuzzy number[4]}

A Generalized Type-2 Hexagonal fuzzy number $\bar{A}$ is defined by

$A=\left\{\left(x, \mu_{A}^{L}(x), \mu_{A}^{M}(x), \mu_{A}^{N}(x), \mu_{A}^{p}(x), \mu_{A}^{Q}(x), \mu_{A}^{R}(x)\right)\right\}$ and $\mu_{A}^{L}(x) \leq \mu_{A}^{M}(x) \leq \mu_{A}^{N}(x) \leq \mu_{A}^{P}(x) \leq \mu_{A}^{Q}(x) \leq \mu_{A}^{R}(x)$ for all $x \in \mathrm{R}$.

It is denoted by

$A=\left(\left(a_{1}^{L}, a_{2}^{L}, a_{1}^{L}, a_{4}^{L}, a_{5}^{L}, a_{6}^{L} ; \lambda_{A}\right)\right.$, $\left(a_{1}^{M}, a_{2}^{M}, a_{9}^{M}, a_{4}^{M}, a_{5}^{M}, a_{6}^{M} ; \sigma_{A}\right),\left(a_{1}^{N}, a_{2}^{N}, a_{2}^{N}, a_{4}^{N}, a_{5}^{N}, a_{6}^{N}, \tau_{A}\right)$, $\left(a_{1}^{p}, a_{2}^{p}, a_{1}^{p}, a_{4}^{p}, a_{5}^{p}, a_{6}^{p}, p_{A}\right),\left(a_{1}^{Q}, a_{2}^{Q}, a_{2}^{Q}, a_{4}^{Q}, a_{5}^{Q}, a_{6}^{Q}, a_{A}\right)$, $\left.\left(a_{1}^{R}, a_{2}^{R}, a_{9}^{R}, a_{4}^{R}, a_{5}^{R}, a_{6}^{R} ; \gamma_{A}\right) ; \omega_{A}\right)$

\section{F. Generalized type-2 octagonal fuzzy number}

A Generalized Type-2 Octagonal fuzzy number $A$ is defined by

$A=\left\{\left(x, \mu_{A}^{L}(x), \mu_{A}^{M}(x), \mu_{A}^{N}(x), \mu_{A}^{P}(x), \mu_{A}^{Q}(x), \mu_{A}^{R}(x)\right.\right.$,

$\left.\left.\mu_{A}^{5}(x), \mu_{A}^{T}(x)\right)\right\}$ and

$\mu_{A}^{L}(x) \leq \mu_{A}^{M}(x) \leq \mu_{A}^{N}(x) \leq \mu_{A}^{p}(x) \leq \mu_{A}^{Q}(x) \leq$

$\mu_{A}^{R}(x) \leq \mu_{A}^{S}(x) \leq \mu_{A}^{T}(x)$ for all $x \in \mathrm{R}$.

It is denoted by

$A=\left(\left(a_{1}^{L}, a_{2}^{L}, a_{2}^{L}, a_{4}^{L}, a_{5}^{L}, a_{6}^{L}, a_{7}^{L}, a_{9}^{L} ; \lambda_{A}\right)\right.$,

$\left(a_{1}^{M}, a_{2}^{M}, a_{2}^{M}, a_{4}^{M}, a_{5}^{M}, a_{6}^{M}, a_{7}^{M}, a_{g}^{M} ; \sigma_{A}\right)$,

$\left(a_{1}^{N}, a_{2}^{N}, a_{\mathrm{g}}^{N}, a_{4}^{N}, a_{5}^{N}, a_{6}^{N}, a_{7}^{N}, a_{9}^{N} ; \tau_{A}\right)$,

$\left(a_{1}^{p}, a_{2}^{p}, a_{9}^{p}, a_{4}^{p}, a_{5}^{p}, a_{6}^{p}, a_{7}^{p}, a_{g}^{p} ; p_{A}\right)$,

$\left(a_{1}^{Q}, a_{2}^{Q}, a_{3}^{Q}, a_{4}^{Q}, a_{5}^{Q}, a_{6}^{Q}, a_{7}^{Q}, a_{8}^{Q}, a_{A}\right)$,

$\left(a_{1}^{R}, a_{2}^{R}, a_{7}^{R}, a_{4}^{R}, a_{5}^{R}, a_{6}^{R}, a_{7}^{R}, a_{9}^{R} ; \beta_{A}\right)$,

$\left(a_{1}^{5}, a_{2}^{5}, a_{2,}^{5}, a_{4,}^{5}, a_{5}^{5}, a_{6,}^{5}, a_{7}^{5}, a_{9}^{5}, \gamma_{A}\right)$,

$\left.\left(a_{1}^{T}, a_{2}^{T}, a_{1}^{T}, a_{4}^{T}, a_{5}^{T}, a_{6}^{T}, a_{7}^{T}, a_{g}^{T} ; \delta_{A}\right) ; \omega_{A}\right)$

\section{G. Properties of Generalized type-2 octagonal fuzzy} number

Let $A=\left(\left(a_{1}^{L}, a_{2}^{L}, a_{1}^{L}, a_{4}^{L}, a_{5}^{L}, a_{6}^{L}, a_{7}^{L}, a_{2}^{L} ; \lambda_{A}\right)\right.$, $\left(a_{1}^{M}, a_{2}^{M}, a_{3}^{M}, a_{4}^{M}, a_{5}^{M}, a_{6}^{M}, a_{7}^{M}, a_{8}^{M} ; \sigma_{A}\right)$,

$\left(a_{1}^{N}, a_{2}^{N}, a_{\mathrm{a}}^{N}, a_{4}^{N}, a_{5}^{N}, a_{6}^{N}, a_{7}^{N}, a_{8}^{N} ; \tau_{A}\right)$,

$\left(a_{1}^{p}, a_{2}^{p}, a_{2}^{p}, a_{4}^{p}, a_{5}^{p}, a_{6}^{p}, a_{7}^{p}, a_{8}^{p} ; p_{A}\right)$,

$\left(a_{1}^{Q}, a_{2}^{Q}, a_{1}^{Q}, a_{4}^{Q}, a_{5}^{Q}, a_{6}^{Q}, a_{7}^{Q}, a_{\mathrm{g}}^{Q}, \alpha_{A}\right)$,

$\left(a_{1}^{R}, a_{2}^{R}, a_{1}^{R}, a_{4}^{R}, a_{5}^{R}, a_{6}^{R}, a_{7}^{R}, a_{g}^{R} ; \beta_{A}\right)$,

$\left(a_{1}^{5}, a_{2}^{5}, a_{2}^{5}, a_{4,}^{5}, a_{5}^{5}, a_{6,}^{5}, a_{7}^{5}, a_{9}^{5} ; \gamma_{A}\right)$,

$\left.\left(a_{1}^{T}, a_{2}^{T}, a_{2}^{T}, a_{4}^{T}, a_{5}^{T}, a_{6}^{T}, a_{7}^{T}, a_{g}^{T} ; \delta_{A}\right) ; \omega_{A}\right)$

And $B=\left(\left(b_{1}^{L}, b_{2}^{L}, b_{\mathrm{a}}^{L}, b_{4}^{L}, b_{5}^{L}, b_{6}^{L}, b_{7}^{L}, b_{8}^{L}, \lambda_{B}\right)\right.$, $\left(b_{1}^{M}, b_{2}^{M}, b_{a}^{M}, b_{4}^{M}, b_{5}^{M}, b_{6}^{M}, b_{7}^{M}, b_{9}^{M} ; \sigma_{B}\right)$,

$\left(b_{1}^{N}, b_{2}^{N}, b_{9}^{N}, b_{4}^{N}, b_{5}^{N}, b_{6}^{N}, b_{7}^{N}, b_{g}^{N} ; \tau_{B}\right)$,

$\left(b_{1}^{P}, b_{2}^{P}, b_{2}^{P}, b_{4}^{P}, b_{5}^{P}, b_{6}^{P}, b_{7}^{P}, b_{9}^{P} ; \rho_{B}\right)$,

$\left(b_{1}^{Q}, b_{2}^{Q}, b_{3}^{Q}, b_{4}^{Q}, b_{5}^{Q}, b_{6}^{Q}, b_{7}^{Q}, b_{9}^{Q} ; \alpha_{B}\right)$,

\section{$\left(b_{1}^{R}, b_{2}^{R}, b_{3}^{R}, b_{4}^{R}, b_{5}^{R}, b_{6}^{R}, b_{7}^{R}, b_{\frac{\pi}{R}}^{R}, \beta_{B}\right)$, $\left(b_{1}^{5}, b_{2}^{5}, b_{2}^{5}, b_{4}^{5}, b_{5}^{5}, b_{6}^{5}, b_{7}^{5}, b_{9}^{5}, \gamma_{B}\right)$, \\ $\left.b_{1}^{T}, b_{2}^{T}, b_{1}^{T}, b_{4}^{T}, b_{5}^{T}, b_{6}^{T}, b_{7}^{T}, b_{\frac{8}{T}}^{T}, \delta_{B}\right)$ ; $\left.\omega_{B}\right)$}

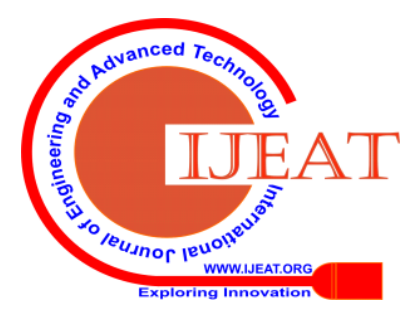


be two generalized type-2 octagonal fuzzy numbers.

Then,

\section{Addition $\oplus$ :}

$\bar{A} \oplus B=\left(\left(a_{1}^{L}+b_{1}^{L}, a_{2}^{L}+b_{2}^{L}, a_{2}^{L}+b_{2}^{L}, a_{4}^{L}+b_{4}^{L}, a_{5}^{L}+b_{5}^{L}\right.\right.$, $\left.a_{6}^{L}+b_{6}^{L}, a_{7}^{L}+b_{7}^{L}, a_{9}^{L}+b_{9}^{L}\right),\left(a_{1}^{M}+b_{1}^{M}, a_{2}^{M}+b_{2}^{M}, a_{a}^{M}+b_{a}^{M}\right.$, $\left.a_{4}^{M}+b_{4}^{M}, a_{5}^{M}+b_{5}^{M}, a_{6}^{M}+b_{6}^{M}, a_{7}^{M}+b_{7}^{M}, a_{9}^{M}+b_{9}^{M}\right)$,

$\left(a_{1}^{N}+b_{1}^{N}, a_{2}^{N}+b_{2}^{N}, a_{2}^{N}+b_{3}^{N}, a_{4}^{N}+b_{4}^{N}, a_{5}^{N}+b_{5}^{N}, a_{6}^{N}+b_{6}^{N}\right.$, $\left.a_{7}^{N}+b_{7}^{N}, a_{g}^{N}+b_{g}^{N}\right),\left(a_{1}^{p}+b_{1}^{p}, a_{2}^{p}+b_{2}^{p}, a_{2}^{p}+b_{3}^{p}, a_{4}^{p}+b_{4}^{p}\right.$, $\left.a_{5}^{p}+b_{5}^{p}, a_{6}^{p}+b_{6}^{p}, a_{7}^{p}+b_{7}^{p}, a_{g}^{p}+b_{8}^{p}\right),\left(a_{1}^{Q}+b_{1}^{q}, a_{2}^{Q}+b_{2}^{Q}\right.$, $\left.a_{3}^{Q}+b_{3}^{Q}, a_{4}^{Q}+b_{4}^{Q}, a_{5}^{Q}+b_{5}^{Q}, a_{6}^{Q}+b_{6}^{Q}, a_{7}^{Q}+b_{7}^{Q}, a_{8}^{Q}+b_{9}^{Q}\right)$, $\left(a_{1}^{R}+b_{1}^{R}, a_{2}^{R}+b_{2}^{R}, a_{2}^{R}+b_{3}^{R}, a_{4}^{R}+b_{4}^{R}, a_{5}^{R}+b_{5}^{R}, a_{6}^{R}+b_{6}^{R} x\right.$ $\left.a_{7}^{R}+b_{7}^{R}, a_{g}^{R}+b_{9}^{R}\right),\left(a_{1}^{5}+b_{1}^{S}, a_{2}^{5}+b_{2}^{5}, a_{2}^{5}+b_{2}^{5}, a_{4}^{5}+b_{4}^{5}\right.$ $\left.a_{5}^{5}+b_{5}^{5}, a_{6}^{5}+b_{6}^{5}, a_{7}^{5}+b_{7}^{5}, a_{g}^{5}+b_{g}^{5}\right),\left(a_{1}^{T}+b_{1}^{T}, a_{2}^{T}+b_{2}^{T}\right.$, $\left.\left.a_{a}^{T}+b_{9}^{T}, a_{4}^{T}+b_{4}^{T}, a_{5}^{T}+b_{5}^{T}, a_{6}^{T}+b_{6}^{T} \times a_{7}^{T}+b_{7}^{T}, a_{9}^{T}+b_{9}^{T}\right)\right)$

Subtraction $\Theta$ :

$\vec{A} \theta \vec{B}=\left(\left(\max \left(0, a_{1}^{L}-b_{9}^{L}\right), \max \left(0, a_{2}^{L}-b_{7}^{L}\right)\right.\right.$, $\max \left(0, a_{a}^{L}-b_{6}^{L}\right), \max \left(0, a_{4}^{L}-b_{5}^{L}\right), \max \left(0, a_{5}^{L}-b_{4}^{L}\right)$, $\left.\max \left(0, a_{6}^{L}-b_{7}^{L}\right), \max \left(0, a_{7}^{L}-b_{2}^{L}\right), \max \left(0, a_{g}^{L}-b_{1}^{L}\right)\right)$, $\left(\max \left(0, a_{1}^{M}-b_{8}^{M}\right), \max \left(0, a_{2}^{M}-b_{7}^{M}\right), \max \left(0, a_{\mathrm{g}}^{M}-b_{6}^{M}\right)\right.$, $\max \left(0, a_{4}^{M}-b_{5}^{M}\right), \max \left(0, a_{5}^{M}-b_{4}^{M}\right), \max \left(0, a_{6}^{M}-b_{9}^{M}\right)$, $\left.\max \left(0, a_{7}^{M}-b_{2}^{M}\right), \max \left(0, a_{g}^{M}-b_{1}^{M}\right)\right),\left(\max \left(0, a_{1}^{N}-b_{g}^{M}\right)\right.$, $\max \left(0, a_{2}^{N}-b_{7}^{N}\right), \max \left(0, a_{a}^{N}-b_{6}^{N}\right), \max \left(0, a_{4}^{N}-b_{5}^{N}\right)$, $\max \left(0, a_{5}^{N}-b_{4}^{N}\right), \max \left(0, a_{6}^{N}-b_{a}^{N}\right), \max \left(0, a_{7}^{N}-b_{2}^{N}\right)$, $\left.\max \left(0, a_{g}^{N}-b_{1}^{N}\right)\right),\left(\max \left(0, a_{1}^{p}-b_{9}^{p}\right), \max \left(0, a_{2}^{p}-b_{7}^{p}\right)\right.$, $\max \left(0, a_{a}^{p}-b_{6}^{p}\right), \max \left(0, a_{4}^{p}-b_{5}^{p}\right), \max \left(0, a_{5}^{p}-b_{4}^{p}\right)$, $\left.\max \left(0, a_{6}^{p}-b_{a}^{p}\right), \max \left(0, a_{7}^{p}-b_{2}^{p}\right), \max \left(0, a_{9}^{p}-b_{1}^{p}\right)\right)$, $\left(\max \left(0, a_{1}^{Q}-b_{0}^{Q}\right), \max \left(0, a_{2}^{Q}-b_{7}^{Q}\right), \max \left(0, a_{7}^{Q}-b_{6}^{Q}\right)\right.$, $\max \left(0, a_{4}^{Q}-b_{5}^{Q}\right), \max \left(0, a_{5}^{Q}-b_{4}^{Q}\right), \max \left(0, a_{6}^{Q}-b_{3}^{Q}\right)$, $\left.\max \left(0, a_{7}^{Q}-b_{2}^{Q}\right), \max \left(0, a_{9}^{Q}-b_{1}^{Q}\right)\right),\left(\max \left(0, a_{1}^{R}-b_{8}^{R}\right)\right.$, $\max \left(0, a_{2}^{R}-b_{7}^{R}\right), \max \left(0, a_{a}^{R}-b_{6}^{R}\right), \max \left(0, a_{4}^{R}-b_{5}^{R}\right)$, $\max \left(0, a_{5}^{R}-b_{4}^{R}\right), \max \left(0, a_{6}^{R}-b_{7}^{R}\right), \max \left(0, a_{7}^{R}-b_{2}^{R}\right)$, $\left.\max \left(0, a_{g}^{R}-b_{1}^{R}\right)\right),\left(\max \left(0, a_{1}^{S}-b_{g}^{5}\right), \max \left(0, a_{2}^{5}-b_{7}^{5}\right)\right.$, $\max \left(0, a_{1}^{5}-b_{6}^{5}\right), \max \left(0, a_{4}^{5}-b_{5}^{5}\right), \max \left(0, a_{5}^{5}-b_{4}^{5}\right)$, $\left.\max \left(0, a_{6}^{5}-b_{2}^{5}\right), \max \left(0, a_{7}^{5}-b_{2}^{5}\right), \max \left(0, a_{g}^{5}-b_{1}^{5}\right)\right)$, $\left(\max \left(0, a_{1}^{T}-b_{g}^{T}\right), \max \left(0, a_{2}^{T}-b_{7}^{T}\right), \max \left(0, a_{2}^{T}-b_{6}^{T}\right)\right.$, $\max \left(0, a_{4}^{T}-b_{5}^{T}\right), \max \left(0, a_{5}^{T}-b_{4}^{T}\right), \max \left(0, a_{6}^{T}-b_{9}^{T}\right)$, $\left.\left.\max \left(0, a_{7}^{T}-b_{2}^{T}\right), \max \left(0, a_{g}^{T}-b_{1}^{T}\right)\right)\right)$

\section{Multiplication}

$A \times B=\left(\left(\frac{a_{1}^{L}}{64} \sigma_{b}, \frac{a_{2}^{L}}{64} \sigma_{b}, \frac{a_{3}^{L}}{64} \sigma_{b x} \frac{a_{4}^{L}}{64} \sigma_{b x} \frac{a_{5}^{L}}{64} \sigma_{b}, \frac{a_{6}^{\frac{L}{6}}}{64} \sigma_{b x} \frac{a_{7}^{\frac{L}{6}}}{64} \sigma_{b x}\right.\right.$ $\left.\frac{a_{B}^{L}}{64} \sigma_{b} ; \min \left(\lambda_{A}, \lambda_{B}\right)\right),\left(\frac{a_{1}^{M}}{64} \sigma_{b}, \frac{a_{2}^{M}}{64} \sigma_{b x} \frac{a_{3}^{M}}{64} \sigma_{b}, \frac{a_{4}^{M}}{64} \sigma_{b}, \frac{a_{5}^{M}}{64} \sigma_{b x}\right.$ $\left.\frac{a_{E}^{M}}{64} \sigma_{b x} \frac{a_{7}^{M}}{64} \sigma_{b x} \frac{a_{B}^{M}}{64} \sigma_{b} ; \min \left(\sigma_{A^{x}} \sigma_{B}\right)\right),\left(\frac{a_{1}^{N}}{64} \sigma_{b x} \frac{a_{2}^{N}}{64} \sigma_{b} \frac{\frac{a_{3}^{N}}{64}}{6} \sigma_{b}\right.$, $\left.\frac{a_{4}^{N}}{64} \sigma_{b}, \frac{a_{5}^{N}}{64} \sigma_{b x} \frac{a_{E}^{N}}{64} \sigma_{b x} \frac{a_{7}^{N}}{64} \sigma_{b}, \frac{a_{B}^{N}}{64} \sigma_{b}: \min \left(\tau_{A}, \tau_{B}\right)\right),\left(\frac{a_{1}^{P}}{64} \sigma_{b}\right.$, $\left.\frac{a_{2}^{F}}{64} \sigma_{b} \frac{a_{2}^{P}}{64} \sigma_{b x} \frac{a_{4}^{P}}{64} \sigma_{b x} \frac{a_{5}^{P}}{64} \sigma_{b x} \frac{a_{b}^{P}}{64} \sigma_{b}, \frac{a_{F}^{P}}{64} \sigma_{b x} \frac{a_{p}^{P}}{64} \sigma_{b} \min \left(\rho_{A}, \rho_{B}\right)\right)$, $\left(\frac{a_{1}^{Q}}{64} \sigma_{b}, \frac{a_{2}^{Q}}{64} \sigma_{b}, \frac{a_{3}^{Q}}{64} \sigma_{b x} \frac{a_{4}^{Q}}{64} \sigma_{b x} \frac{a_{5}^{Q}}{64} \sigma_{b}, \frac{a_{6}^{Q}}{64} \sigma_{b x} \frac{a_{7}^{Q}}{64} \sigma_{b x} \frac{a_{8}^{Q}}{64} \sigma_{b}\right.$ $\left.\min \left(\alpha_{A}, \alpha_{B}\right)\right),\left(\frac{a_{1}^{R}}{64} \sigma_{b} \frac{a_{2}^{R}}{64} \sigma_{b} \frac{\frac{a_{2}^{R}}{64}}{64} \sigma_{b} \frac{\frac{a_{4}^{R}}{64}}{\sigma_{b} x} \frac{a_{5}^{R}}{64} \sigma_{b}, \frac{\frac{a_{E}^{R}}{64}}{\sigma_{b}}\right.$

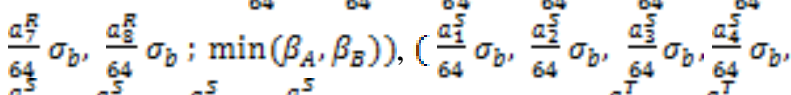
$\left.\frac{a_{5}^{5}}{64} \sigma_{b x} \frac{a_{6}^{5}}{64} \sigma_{b}, \frac{a_{7}^{5}}{64} \sigma_{b x} \frac{a_{g}^{5}}{64} \sigma_{b} ; \min \left(\gamma_{A}, \gamma_{B}\right)\right),\left(\frac{a_{1}^{T}}{64} \sigma_{b x} \frac{\frac{a_{2}^{T}}{64}}{\sigma_{b}}\right.$

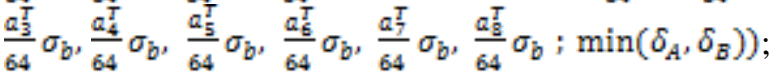
$\left.\min \left(\omega_{A}, \omega_{B}\right)\right)$

Where $\sigma_{b}=b_{1}^{L}+b_{2}^{L}+b_{a}^{L}+b_{4}^{L}+b_{5}^{L}+b_{6}^{L}+b_{7}^{L}+b_{9}^{L}+$ $b_{1}^{M}+b_{2}^{M}+b_{a}^{M}+b_{4}^{M}+b_{5}^{M}+b_{6}^{M}+b_{7}^{M}+b_{9}^{M}+b_{1}^{M}+b_{2}^{M}+$ $b_{a}^{N}+b_{4}^{N}+b_{5}^{N}+b_{6}^{N}+b_{7}^{N}+b_{0}^{N}+b_{1}^{P}+b_{2}^{P}+b_{9}^{P}+b_{4}^{P}+b_{5}^{p}+$ $b_{6}^{P}+b_{7}^{P}+b_{9}^{P}+b_{1}^{Q}+b_{2}^{Q}+b_{3}^{Q}+b_{4}^{Q}+b_{5}^{Q}+b_{6}^{Q}+b_{7}^{Q}+$
$b_{9}^{Q}+b_{1}^{R}+b_{2}^{R}+b_{2}^{R}+b_{4}^{R}+b_{5}^{R}+b_{6}^{R}+b_{7}^{R}+b_{8}^{R}+b_{1}^{S}+b_{2}^{S}+$ $b_{1}^{5}+b_{4}^{S}+b_{5}^{S}+b_{6}^{S}+b_{7}^{S}+b_{9}^{S}+b_{1}^{T}+b_{2}^{T}+b_{1}^{T}+b_{4}^{T}+$ $b_{5}^{T}+b_{6}^{T}+b_{7}^{T}+b_{8}^{T}$

\section{Division}

$A \div B=\left(\left(64 \frac{a_{1}^{L}}{\sigma_{b}}, 64 \frac{a_{2}^{L}}{\sigma_{b}}, 64 \frac{a_{5}^{L}}{\sigma_{b}}, 64 \frac{a_{4}^{L}}{\sigma_{b}}, 64 \frac{a_{5}^{L}}{\sigma_{b}}, 64 \frac{a_{b}^{L}}{\sigma_{b}}\right.\right.$

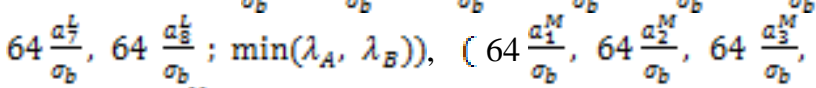
$\left.64 \frac{a_{4}^{M}}{\sigma_{b}}, 64 \frac{a_{5}^{M}}{\sigma_{b}}, 64 \frac{a_{k}^{M}}{\sigma_{b}}, 64 \frac{a_{7}^{M}}{\sigma_{b}}, 64 \frac{a_{B}^{M}}{\sigma_{b}} ; \min \left(\sigma_{A}, \sigma_{B}\right)\right)$, $\left(\frac{a_{1}^{N}}{64} \sigma_{b}, \frac{a_{2}^{N}}{64} \sigma_{b}, \frac{a_{3}^{N}}{64} \sigma_{b}, \frac{a_{4}^{N}}{64} \sigma_{b}, \frac{a_{5}^{N}}{64} \sigma_{b}, \frac{a_{b}^{N}}{64} \sigma_{b}, \frac{a_{7}^{N}}{64} \sigma_{b}, \frac{a_{l}^{N}}{64} \sigma_{b}\right.$;

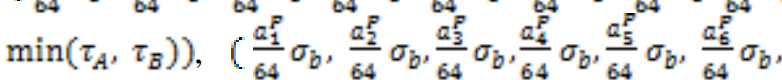
$\left.\frac{a_{7}^{P}}{64} \sigma_{b}, \frac{a_{a}^{P}}{64} \sigma_{b} ; \min \left(\rho_{A}, \rho_{B}\right)\right),\left(\frac{a_{1}^{Q}}{64} \sigma_{b}, \frac{a_{2}^{Q}}{64} \sigma_{b}, \frac{a_{3}^{Q}}{64} \sigma_{b} x \frac{a_{64}^{Q}}{64} \sigma_{b} x\right.$ $\left.\frac{a_{E}^{Q}}{64} \sigma_{b}, \frac{a_{G}^{Q Q}}{64} \sigma_{b}, \frac{a_{7}^{Q}}{64} \sigma_{b}, \frac{a_{R}^{Q}}{64} \sigma_{b} ; \min \left(\alpha_{A}, \alpha_{B}\right)\right),\left(\frac{a_{1}^{R}}{64} \sigma_{b}, \frac{a_{2}^{R}}{64} \sigma_{b x}\right.$

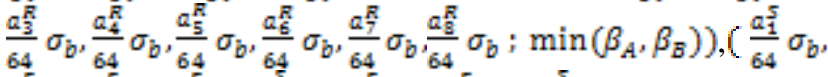
$\left.\frac{a_{2}^{5}}{64} \sigma_{b b} \frac{a_{5}^{5}}{64} \sigma_{b}, \frac{a_{4}^{5}}{64} \sigma_{b}, \frac{a_{5}^{5}}{64} \sigma_{b x} \frac{a_{B}^{5}}{64} \sigma_{b}, \frac{a_{y}^{5}}{64} \sigma_{b}, \frac{a_{a}^{5}}{64} \sigma_{b} ; \min \left(\gamma_{A}, \gamma_{B}\right)\right)$ $\left(\frac{a_{1}^{T}}{64} \sigma_{b} x \frac{a_{2}^{T}}{64} \sigma_{b} x \frac{a_{5}^{T}}{64} \sigma_{b x} \frac{a_{4}^{T}}{64} \sigma_{b} x \frac{a_{5}^{T}}{64} \sigma_{b} x \frac{a_{b}^{T}}{64} \sigma_{b} x \frac{a_{y}^{T}}{64} \sigma_{b} x \frac{a_{a}^{T}}{64} \sigma_{b}\right.$; $\left.\left.\min \left(\delta_{A}, \delta_{B}\right)\right) ; \min \left(\omega_{A}, \omega_{B}\right)\right)$

Where $\sigma_{b}=b_{1}^{L}+b_{2}^{L}+b_{a}^{L}+b_{4}^{L}+b_{5}^{L}+b_{6}^{L}+b_{7}^{L}+b_{9}^{L}+$ $b_{1}^{M}+b_{2}^{M}+b_{2}^{M}+b_{4}^{M}+b_{5}^{M}+b_{6}^{M}+b_{7}^{M}+b_{9}^{M}+b_{1}^{M}+b_{2}^{N}+$ $b_{a}^{N}+b_{4}^{N}+b_{5}^{N}+b_{6}^{N}+b_{7}^{N}+b_{0}^{N}+b_{1}^{p}+b_{2}^{p}+b_{3}^{p}+b_{4}^{p}+b_{5}^{p}+$ $b_{6}^{p}+b_{7}^{p}+b_{9}^{p}+b_{1}^{Q}+b_{2}^{Q}+b_{1}^{Q}+b_{4}^{Q}+b_{5}^{Q}+b_{6}^{Q}+b_{7}^{Q}+$ $b_{9}^{Q}+b_{1}^{R}+b_{2}^{R}+b_{1}^{R}+b_{4}^{R}+b_{5}^{R}+b_{6}^{R}+b_{7}^{R}+b_{0}^{R}+b_{1}^{S}+b_{2}^{S}+$ $b_{a}^{5}+b_{4}^{S}+b_{5}^{S}+b_{6}^{S}+b_{7}^{S}+b_{9}^{S}+b_{1}^{T}+b_{2}^{T}+b_{1}^{T}+b_{4}^{T}+$ $b_{5}^{T}+b_{6}^{T}+b_{7}^{T}+b_{8}^{T}$

\section{Scalar Multiplication}

If $\mathrm{k} \neq 0$ is a scalar, then

$\mathrm{k} A=\left(\left(\left(k a_{1}^{L}, k a_{2}^{L}, k a_{1}^{L}, k a_{4}^{L}, k a_{5}^{L}, k a_{6}^{L}, k a_{7}^{L}, k a_{2}^{L}\right)\right.\right.$, $\left(k a_{1}^{M}, k a_{2}^{M}, k a_{3}^{M}, k a_{4}^{M}, k a_{5}^{M}, k a_{6}^{M}, k a_{7}^{M}, k a_{8}^{M}\right)$, $\left(k a_{1}^{N}, k a_{2}^{N}, k a_{2}^{N}, k a_{4}^{N}, k a_{5}^{N}, k a_{6}^{N}, k a_{7}^{N}, k a_{2}^{N}\right)$, $\left(k a_{1}^{p}, k a_{2}^{p}, k a_{2}^{p}, k a_{4}^{P}, k a_{5}^{p}, k a_{6}^{p}, k a_{7}^{p}, k a_{9}^{p}\right)$, $\left(k a_{1}^{Q}, k a_{2}^{Q}, k a_{3}^{Q}, k a_{4}^{Q}, k a_{5}^{Q}, k a_{6}^{Q}, k a_{7}^{Q}, k a_{0}^{Q}\right)$, $\left(k a_{1}^{R}, k a_{2}^{R}, k a_{2}^{R}, k a_{4}^{R}, k a_{5}^{R}, k a_{6}^{R}, k a_{7}^{R}, k a_{g}^{R}\right)$, $\left(k a_{1}^{5}, k a_{2}^{5}, k a_{2}^{5}, k a_{4}^{5}, k a_{5}^{5}, k a_{6}^{5}, k a_{7}^{5}, k a_{9}^{5}\right)$, $\left.\left.\left(k a_{1}^{T}, k a_{2}^{T}, k a_{1}^{T}, k a_{4}^{T}, k a_{5}^{T}, k a_{6}^{T}, k a_{7}^{T}, k a_{g}^{T}\right)\right) ; \omega_{A}\right)$ if $\mathrm{k}>$ 0.

$\mathrm{k} A=\left(\left(\left(k a_{\mathrm{g}}^{L}, k a_{7}^{L}, k a_{6}^{L}, k a_{5}^{L}, k a_{4}^{L}, k a_{1}^{L}, k a_{2}^{L}, k a_{1}^{L}\right)\right.\right.$, $\left(k a_{8}^{M}, k a_{7}^{M}, k a_{6}^{M}, k a_{5}^{M}, k a_{4}^{M}, k a_{1}^{M}, k a_{2}^{M}, k a_{1}^{M}\right)$, $\left(k a_{2}^{N}, k a_{7}^{N}, k a_{6}^{N}, k a_{5}^{N}, k a_{4}^{N}, k a_{2}^{N}, k a_{2}^{N}, k a_{1}^{N}\right)$, $\left(k a_{9}^{p}, k a_{7}^{p}, k a_{6}^{p}, k a_{5}^{p}, k a_{4}^{p}, k a_{2}^{p}, k a_{2}^{p}, k a_{1}^{p}\right)$, $\left(k a_{0}^{Q}, k a_{7}^{Q}, k a_{6}^{Q}, k a_{5}^{Q}, k a_{4}^{Q}, k a_{3}^{Q}, k a_{2}^{Q}, k a_{1}^{Q}\right)$, $\left(k a_{2}^{R}, k a_{7}^{R}, k a_{6}^{R}, k a_{5}^{R}, k a_{4}^{R}, k a_{2}^{R}, k a_{2}^{R}, k a_{1}^{R}\right)$, $\left(k a_{3}^{5}, k a_{7}^{5}, k a_{6}^{5}, k a_{5}^{5}, k a_{4}^{5}, k a_{2}^{5}, k a_{2}^{5}, k a_{1}^{5}\right)$,

$\left.\left.\left(k a_{g}^{T}, k a_{7}^{T}, k a_{6}^{T}, k a_{5}^{T}, k a_{4}^{T}, k a_{2}^{T}, k a_{2}^{T}, k a_{1}^{T}\right)\right) ; \omega_{A}\right)$ if $\mathrm{k}<0$.

\section{Square Root}

$\sqrt{A}=\left(\left(\sqrt{a_{1}^{L}}, \sqrt{a_{2}^{L}}, \sqrt{a_{\mathrm{a}}^{L}}, \sqrt{a_{4}^{L}}, \sqrt{a_{5}^{L}}, \sqrt{a_{6}^{L}}, \sqrt{a_{7}^{L}}, \sqrt{a_{\mathrm{g}}^{L}}\right)\right.$, $\left(\sqrt{a_{1}^{M}}, \sqrt{a_{2}^{M}}, \sqrt{a_{3}^{M}}, \sqrt{a_{4}^{M}}, \sqrt{a_{5}^{M}}, \sqrt{a_{6}^{M}}, \sqrt{a_{7}^{M}}, \sqrt{a_{8}^{M}}\right)$, $\left(\sqrt{a_{1}^{N}}, \sqrt{a_{2}^{N}}, \sqrt{a_{3}^{N}}, \sqrt{a_{4}^{N}}, \sqrt{a_{5}^{N}}, \sqrt{a_{6}^{N}}, \sqrt{a_{7}^{N}}, \sqrt{a_{9}^{N}}\right)$, $\left(\sqrt{a_{1}^{p}}, \sqrt{a_{2}^{p}}, \sqrt{a_{9}^{p}}, \sqrt{a_{4}^{p}}, \sqrt{a_{5}^{p}}, \sqrt{a_{6}^{p}}, \sqrt{a_{7}^{p}}, \sqrt{a_{9}^{p}}\right)$, $\left(\sqrt{a_{1}^{Q}}, \sqrt{a_{2}^{Q}}, \sqrt{a_{a}^{Q}}, \sqrt{a_{4}^{Q}}, \sqrt{a_{5}^{Q}}\right.$ $\left., \sqrt{a_{6}^{Q}}, \sqrt{a_{7}^{Q}}, \sqrt{a_{g}^{Q}}\right)$,

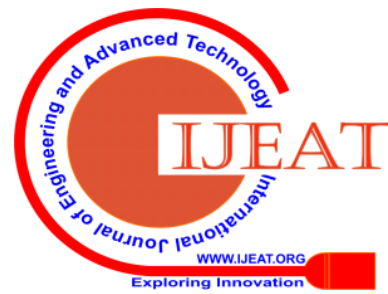




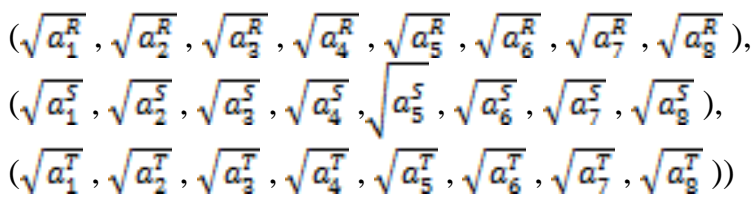

\section{PROPOSED METHODS FOR RANKING OF VARIOUS GENERALIZED TYPE-2 FUZZY NUMBERS}

\section{A. Proposed ranking method of Generalized Type-2} Trapezoidal fuzzy number

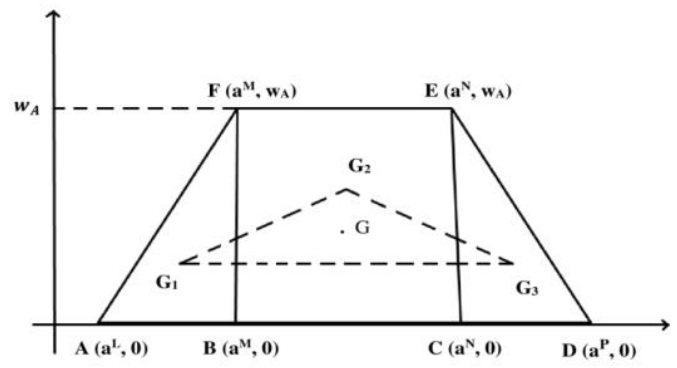

Fig 1. Centroid of Generalized Type-2 Trapezoidal Fuzzy Number

Centroid is the point of centre of gravity of any type of plane figure. The above plane figure is considered as the graphical representation of Generalized Type-2 Trapezoidal Fuzzy Number. In the plane Fig 1, the $\mathrm{x}$ co-ordinates $\mathrm{a}^{\mathrm{L}}, \mathrm{a}^{\mathrm{M}}$, $\mathrm{a}^{\mathrm{N}}$ and $\mathrm{a}^{\mathrm{P}}$ of vertices are considered as centroid of centroids of each trapezoidal fuzzy quantities occurred in the Generalized Type-2 Fuzzy Number. First, the trapezoid is divided into three plane figures ABF (triangle), BCEF (rectangle) and CDE (triangle). The centroid of the centroids of the three plane figures is considered as the center of gravity point of generalized type-2 trapezoidal fuzzy number to define its ranking function. The reason for selecting this point as a center point of gravity is that each Centroid point ( $G_{1}$ of triangle ABF, $G_{2}$ of rectangle BCEF, and $G_{3}$ of triangle CDE) are center of gravity points of each individual plane figure. Therefore, the centroid of these gravity points might be a better equidistant point rather than centroid of trapezoid.

Consider the Generalized Type-2 trapezoidal fuzzy number

$\bar{A}=\left(\left(a_{1}^{L}, a_{2}^{L}, a_{\mathrm{a}}^{L}, a_{4}^{L} ; \lambda_{A}\right),\left(a_{1}^{M}, a_{2}^{M}, a_{\mathrm{a}}^{M}, a_{4}^{M} ; \sigma_{A}\right)\right.$,

$\left.\left(a_{1}^{N}, a_{2}^{N}, a_{2}^{N}, a_{4}^{N} ; \tau_{A}\right),\left(a_{1}^{p}, a_{2}^{p}, a_{2}^{p}, a_{4}^{p}, p_{A}\right) ; \omega_{A}\right)$

In the above figure, the x co-ordinate $a^{L}, a^{M}, a^{N}$ and $a^{P}$ are centroid of centroids of the trapezoidal fuzzy numbers

$\left(a_{1}^{L}, a_{2}^{L}, a_{1}^{L}, a_{4}^{L} ; \lambda_{A}\right),\left(a_{1}^{M}, a_{2}^{M}, a_{a}^{M}, a_{4}^{M} ; \sigma_{A}\right)$,

$\left(a_{1}^{N}, a_{2}^{N}, a_{a}^{N}, a_{4}^{N} ; \tau_{A}\right)$ and $\left(a_{1}^{p}, a_{2}^{p}, a_{9}^{p}, a_{4}^{p} ; p_{A}\right)$ respectively.

For the sake of clarity, the centroid points of the three plane figures are presented as follows:

The centroid of $\mathrm{ABC}$ is

$$
\begin{aligned}
G_{1}= & \left(\frac { 1 } { 5 4 } \left\{2\left(a_{1}^{L}+a_{4}^{L}\right)+4\left(a_{1}^{M}+a_{4}^{M}\right)+7\left(a_{2}^{L}+a_{a}^{L}\right)+\right.\right. \\
& \left.\left.14\left(a_{2}^{M}+a_{a}^{M}\right)\right\}, \frac{\omega_{A}}{a}\right)
\end{aligned}
$$

The centroid of BCEF is

$$
\begin{aligned}
G_{2}= & \left(\frac { 1 } { 7 2 } \left\{4\left(a_{1}^{M}+a_{4}^{M}+a_{1}^{N}+a_{4}^{N}\right)+14\left(a_{2}^{M}+a_{a}^{M}+\right.\right.\right. \\
& \left.\left.\left.a_{2}^{N}+a_{a}^{N}\right)\right\}, \frac{\omega_{A}}{2}\right)
\end{aligned}
$$

The centroid of CDE is

$G_{a}=\left(\frac{1}{54}\left\{2\left(a_{1}^{p}+a_{4}^{p}\right)+4\left(a_{1}^{N}+a_{4}^{N}\right)+7\left(a_{2}^{p}+a_{a}^{p}\right)+\right.\right.$

$$
\left.\left.14\left(a_{2}^{N}+a_{a}^{N}\right)\right\}, \frac{\omega_{A}}{a}\right)
$$

Hence the centroid of $G_{1}, G_{2}$ and $G_{2}$ is

$$
\begin{aligned}
\mathrm{G}= & (x(\widetilde{\widetilde{A}}), y(\widetilde{\widetilde{A}}))=\left(\frac { 1 } { a 2 4 } \left\{4\left(a_{1}^{L}+a_{4}^{L}+a_{1}^{p}+a_{4}^{p}\right)+\right.\right. \\
& 14\left(a_{2}^{L}+a_{a}^{L}+a_{2}^{p}+a_{a}^{p}+a_{1}^{M}+a_{4}^{M}+a_{1}^{N}+a_{4}^{M}\right)+ \\
& \left.\left.49\left(a_{2}^{M}+a_{a}^{M}+a_{2}^{N}+a_{a}^{N}\right)\right\}, \frac{7 \omega_{A}}{18}\right)
\end{aligned}
$$

Then the ranking function of $\mathbb{A}$ is defined as

$$
\mathrm{R}(\tilde{A})=x(\stackrel{\widetilde{\widetilde{A}}}{)} \alpha+(1-\alpha) y(\stackrel{\widetilde{\widetilde{A}}}{)}
$$

For all the possible paths, their corresponding path lengths $L_{j}, \mathrm{j}=1,2,3, \ldots \mathrm{n}$ to be fuzzified in an uncertain context where $L_{j}=[\mathrm{b}, \mathrm{c}]$ is considered as a Generalized Type-2 trapezoidal fuzzy number.

$$
\begin{aligned}
\tilde{L}_{j}= & {[\mathrm{b}, \mathrm{c}]=\left(\left(\mathrm{b}-\delta_{j 04}, \mathrm{~b}, \mathrm{c}, \mathrm{c}+\delta_{j 05} ; \lambda_{j}\right),\right.} \\
& \left(\mathrm{b}-\delta_{j 0 \mathrm{a}}, \mathrm{b}, \mathrm{c}, \mathrm{c}+\delta_{j 06} ; \sigma_{j}\right),\left(\mathrm{b}-\delta_{j 02}, \mathrm{~b}, \mathrm{c}, \mathrm{c}+\delta_{j 07} ; \tau_{j}\right), \\
& \left.\left(\mathrm{b}-\delta_{j 01}, \mathrm{~b}, \mathrm{c}, \mathrm{c}+\delta_{j 08} ; \mathrm{P}_{j}\right) ; \omega_{j}\right)
\end{aligned}
$$

Where $0<\delta_{j 04}<\delta_{j 03}<\delta_{j 02}<\delta_{j 01}$ and

$0<\delta_{j 05}<\delta_{j 06}<\delta_{j 07}<\delta_{j 08}$

Implies $\mathrm{b}>\mathrm{b}-\delta_{j 04}>\mathrm{b}-\delta_{j 0 \mathrm{a}}>\mathrm{b}-\delta_{j 02}>\mathrm{b}-\delta_{j 01}$ and

$\mathrm{c}<\mathrm{c}+\delta_{j 05}<\mathrm{c}+\delta_{j 06}<\mathrm{c}+\delta_{j 07}<\mathrm{c}+\delta_{j 08}$

The graphical representation of generalized type-2 trapezoidal fuzzy number defined in equation (3) is shown in the following Fig. 2.

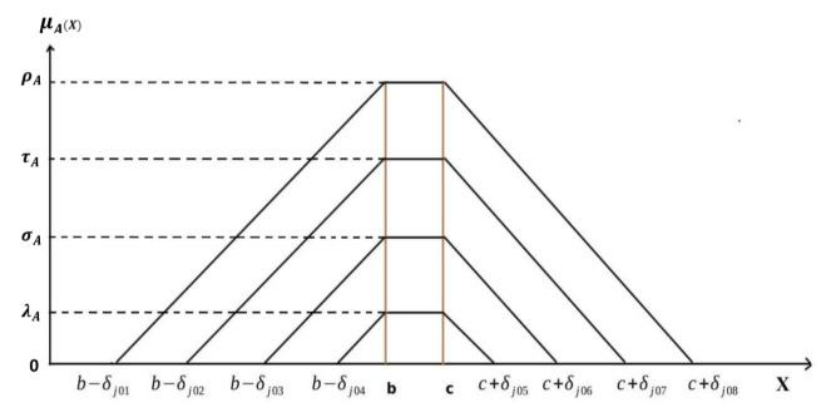

Fig 2. Generalized Type-2 Trapezoidal Fuzzy Number

Therefore the centroid of trapezoidal fuzzy arc lengths is defined by using the proposed centroid of trapezoidal fuzzy number (1) as follows:

$\mathrm{G}=(x(\tilde{\tilde{L}}), y(\tilde{L}))=\left(\frac{1}{162}\left\{81(\mathrm{~b}+\mathrm{c})+2\left(\delta_{j 05}+\delta_{j 08}-\right.\right.\right.$
$\left.\left.\delta_{j 01}-\delta_{j 04}\right)+7\left(\delta_{j 06}+\delta_{j 07}-\delta_{j 02}-\delta_{j 00}\right), \frac{7 \omega_{A}}{18}\right)$

(4)

Therefore the ranking function for all possible arc lengths is defined as:

$$
\mathrm{R}(\tilde{A})=x(\widetilde{\widetilde{L}}) \alpha+(1-\alpha) y(\widetilde{\widetilde{L}})
$$

\section{B. Proposed ranking method of Generalized type-2 hexagonal fuzzy number}

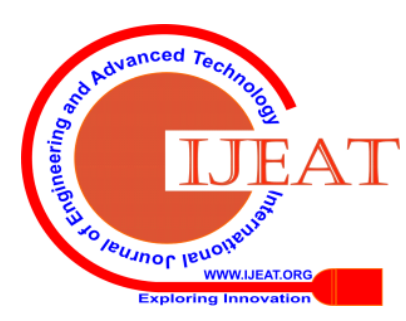




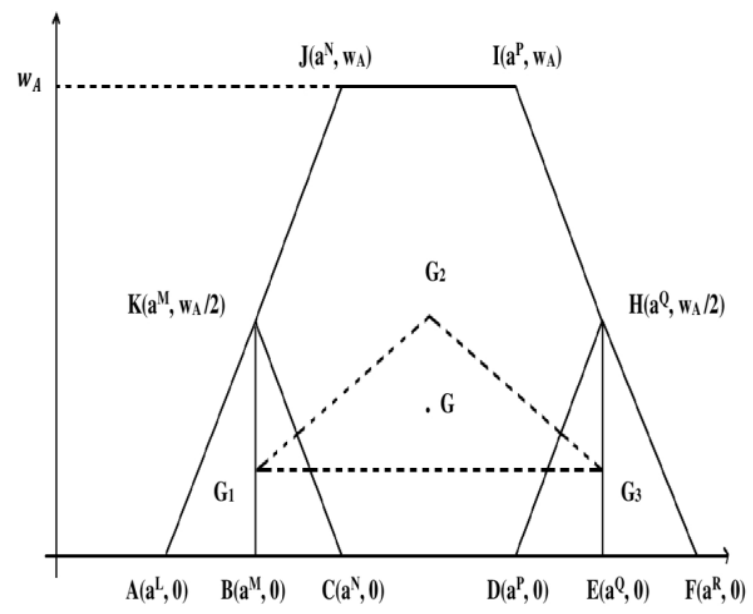

Fig 3. Centroid of Generalized Type-2 Hexagonal Fuzzy Number

Centroid is the point of centre of gravity of any type of plane figure. The above plane figure is considered as the graphical representation of Generalized Type-2 Hexagonal Fuzzy Number. In the plane Fig. 3 , the $\mathrm{x}$ co-ordinates $\mathrm{a}^{\mathrm{L}}, \mathrm{a}^{\mathrm{M}}, \mathrm{a}^{\mathrm{N}}, \mathrm{a}^{\mathrm{P}}$, $\mathrm{a}^{\mathrm{Q}}$ and $\mathrm{a}^{\mathrm{R}}$ of vertices are considered as centroid of centroids of each hexagonal fuzzy quantities occurred in the Generalized Type-2 Hexagonal Fuzzy Number. First, the hexagon is divided into three plane figures ACK (triangle), CDHIJK (hexagon) and DFH (triangle). The centroid of the Centroids of the three plane figures is considered as the center of gravity point of generalized type-2 hexagonal fuzzy number to define its ranking function. The reason for selecting this point as a center point of gravity is that each Centroid point ( $G_{1}$ of triangle ACK, $G_{2}$ of hexagon CDHIJK, and $G_{3}$ of triangle DFH) are center of gravity points of each individual plane figure. Therefore, the centroid of these gravity points might be a better equidistant point rather than centroid of hexagon.

Consider the Generalized Type-2 hexagonal fuzzy number $A=\left(\left(a_{1}^{L}, a_{2}^{L}, a_{1}^{L}, a_{4}^{L}, a_{5}^{L}, a_{6}^{L} ; \lambda_{A}\right),\left(a_{1}^{M}, a_{2}^{M}, a_{3}^{M}, a_{4}^{M}, a_{5}^{M}, a_{6}^{M} ; \sigma_{A}\right)\right.$, $\left(a_{1}^{N}, a_{2}^{N}, a_{1}^{N}, a_{4}^{N}, a_{5}^{N}, a_{6}^{N}, \tau_{A}\right),\left(a_{1}^{p}, a_{2}^{p}, a_{1}^{p}, a_{4}^{p}, a_{5}^{p}, a_{6}^{p}, p_{A}\right)$, $\left.\left(a_{1}^{Q}, a_{2}^{Q}, a_{1}^{Q}, a_{4}^{Q}, a_{5}^{Q}, a_{6}^{Q} ; \alpha_{A}\right),\left(a_{1}^{R}, a_{2}^{R}, a_{1}^{R}, a_{4}^{R}, a_{5}^{R}, a_{6}^{R} ; \gamma_{A}\right) ; w_{A}\right)$

In figure 3 , the $x$ co-ordinates $a^{L}, a^{M}, a^{N}, a^{P}, a^{Q}$ and $a^{R}$ are centroid of centroids of the hexagonal fuzzy numbers $\left(a_{1}^{L}, a_{2}^{L}, a_{2}^{L}, a_{4}^{L}, a_{5}^{L}, a_{6}^{L} ; \lambda_{A}\right),\left(a_{1}^{M}, a_{2}^{M}, a_{2}^{M}, a_{4}^{M}, a_{5}^{M}, a_{6}^{M} ; \sigma_{A}\right)$, $\left(a_{1}^{N}, a_{2}^{N}, a_{1}^{N}, a_{4}^{N}, a_{5}^{N}, a_{6}^{N}, \tau_{A}\right),\left(a_{1}^{p}, a_{2}^{p}, a_{a}^{p}, a_{4}^{p}, a_{5}^{p}, a_{6}^{p} ; \rho_{A}\right)$, $\left(a_{1}^{Q}, a_{2}^{Q}, a_{3}^{Q}, a_{4}^{Q}, a_{5}^{Q}, a_{6}^{Q}, a_{A}\right)$ and $\left(a_{1}^{R}, a_{2}^{R}, a_{3}^{R}, a_{4}^{R}, a_{5}^{R}, a_{6}^{R} ; \gamma_{A}\right)$ respectively. For the sake of clarity, the centroid points of the three plane figures are presented as follows:

The centroid of ACK is

$$
\begin{aligned}
G_{1}= & \left(\frac { 1 } { 5 4 } \left\{2\left(a_{1}^{L}+a_{6}^{L}+a_{1}^{N}+a_{6}^{N}+a_{1}^{M}+a_{6}^{M}\right)+\right.\right. \\
& 3\left(a_{2}^{L}+a_{5}^{L}+a_{2}^{N}+a_{5}^{N}+a_{2}^{M}+a_{5}^{M}\right)+ \\
& \left.\left.4\left(a_{3}^{L}+a_{4}^{L}+a_{a}^{N}+a_{4}^{N}+a_{3}^{M}+a_{4}^{M}\right)\right\}, \frac{\omega_{A}}{6}\right)
\end{aligned}
$$

The centroid of CDHIJK is

$$
\begin{aligned}
& G_{2}=\left(\frac { 1 } { 1 0 8 } \left\{2\left(a_{1}^{Q}+a_{6}^{Q}+a_{1}^{M}+a_{6}^{M}\right)+3\left(a_{2}^{Q}+a_{5}^{Q}\right.\right.\right. \\
&\left.+a_{2}^{M}+a_{5}^{M}\right)+4\left(a_{1}^{N}+a_{6}^{N}+a_{1}^{p}+a_{6}^{p}+\right. \\
&\left.a_{a}^{Q}+a_{4}^{Q}+a_{2}^{M}+a_{4}^{M}\right)+6\left(a_{N}^{N}+a_{5}^{N}+a_{2}^{p}+a_{5}^{p}\right)+ \\
&\left.\left.8\left(a_{a}^{N}+a_{4}^{N}+a_{a}^{p}+a_{4}^{p}\right)\right\}, \frac{3 \omega_{A}}{6}\right)
\end{aligned}
$$

The centroid of DFH is

$$
\begin{aligned}
& G_{a}=\left(\frac { 1 } { 5 4 } \left\{2\left(a_{1}^{p}+a_{6}^{p}+a_{1}^{R}+a_{6}^{R}+a_{1}^{Q}+a_{6}^{Q}\right)+\right.\right. \\
& 3\left(a_{2}^{p}+a_{5}^{p}+a_{2}^{R}+a_{5}^{R}+a_{2}^{Q}+a_{5}^{Q}\right)+ \\
& \left.\left.4\left(a_{a}^{p}+a_{4}^{p}+a_{a}^{R}+a_{4}^{R}+a_{a}^{Q}+a_{4}^{Q}\right)\right\}, \frac{\omega_{A}}{6}\right) \\
& \mathrm{G}=(x(\widetilde{\widetilde{A}}), y(\widetilde{\widetilde{A}})) \\
& =\left(\frac { 1 } { 1 2 4 } \left\{2\left(a_{1}^{M}+a_{6}^{M}+a_{1}^{Q}+a_{6}^{Q}\right)+3\left(a_{2}^{M}+a_{5}^{M}+a_{2}^{Q}+\right.\right.\right. \\
& \left.a_{5}^{Q}\right)+4\left(a_{1}^{L}+a_{6}^{L}+2 a_{1}^{N}+2 a_{6}^{N}+a_{1}^{M}+a_{a}^{M}+a_{4}^{M}+a_{6}^{M}+\right. \\
& \left.2 a_{1}^{p}+2 a_{6}^{p}+a_{1}^{Q}+a_{1}^{Q}+a_{4}^{Q}+a_{6}^{Q}+a_{1}^{R}+a_{6}^{R}\right)+ \\
& 6\left(a_{2}^{L}+a_{5}^{L}+2 a_{2}^{N}+2 a_{5}^{N}+a_{2}^{M}+a_{5}^{M}+2 a_{2}^{p}+2 a_{5}^{P}+\right. \\
& \left.a_{2}^{Q}+a_{5}^{Q}+a_{2}^{R}+a_{5}^{R}\right)+8\left(a_{2}^{L}+a_{4}^{L}+2 a_{a}^{N}+2 a_{4}^{N}+a_{a}^{M}+\right. \\
& \left.\left.\left.a_{4}^{M}+2 a_{a}^{P}+2 a_{4}^{P}+a_{a}^{Q}+a_{4}^{Q}+a_{a}^{R}+a_{4}^{R}\right)\right\}_{0} \frac{5 w_{A}}{18}\right)
\end{aligned}
$$

Then the ranking function of $A$ is defined as

$$
\mathrm{R}(\tilde{A})=x(\stackrel{\widetilde{\widetilde{A}}}{)} \alpha+(1-\alpha) y(\stackrel{\widetilde{\widetilde{A}}}{)}
$$

For all the possible paths, their corresponding path lengths $L_{j}, j=1,2,3, \ldots \mathrm{n}$ to be fuzzified in an uncertain context where $L_{j}=[\mathrm{c}, \mathrm{d}]$ is considered as a generalized type-2 hexagonal fuzzy number [3].

$\tilde{L}_{\mathrm{g}}^{*}=[\mathrm{c}, \mathrm{d}]$

$=\left(\left(\mathrm{c}-\delta_{j 11}, \mathrm{c}-\delta_{j 12}, \mathrm{c}, \mathrm{d}, \mathrm{d}+\delta_{j 12}, \mathrm{~d}+\delta_{j 14} ; \lambda_{\mathrm{j}}\right)\right.$,

$\left(\mathrm{c}-\delta_{j 09}, \mathrm{c}-\delta_{j 10}, \mathrm{c}, \mathrm{d}, \mathrm{d}+\delta_{j 15}, \mathrm{~d}+\delta_{j 16} ; \sigma_{\mathrm{j}}\right)$,

$\left(\mathrm{c}-\delta_{j 07}, \mathrm{c}-\delta_{j 08}, \mathrm{c}, \mathrm{d}, \mathrm{d}+\delta_{j 17}, \mathrm{~d}+\delta_{j 18} ; \tau_{\mathrm{j}}\right)$,

$\left(\mathrm{c}-\delta_{j 05}, \mathrm{c}-\delta_{j 06}, \mathrm{c}, \mathrm{d}, \mathrm{d}+\delta_{j 19}, \mathrm{~d}+\delta_{j 20} ; \rho_{\mathrm{j}}\right)$,

$\left(\mathrm{c}-\delta_{j 01}, \mathrm{c}-\delta_{j 04}, \mathrm{c}, \mathrm{d}, \mathrm{d}+\delta_{j 21}, \mathrm{~d}+\delta_{j 22} ; \alpha_{\mathrm{j}}\right)$,

$\left.\left(\mathrm{c}-\delta_{j 01}, \mathrm{c}-\delta_{j 02}, \mathrm{c}, \mathrm{d}, \mathrm{d}+\delta_{j 2 \mathrm{a}}, \mathrm{d}+\delta_{j 24} ; \gamma_{\mathrm{j}}\right) ; \omega_{\mathrm{j}}\right)$

(8)

Where $0<\delta_{j 12}<\delta_{j 11}<\delta_{j 10}<\delta_{j 09}<\delta_{j 08}<\delta_{j 07}<\delta_{j 06}<\delta_{j 05}<$ $\delta_{j 04}<\delta_{j 03}<\delta_{j 02}<\delta_{j 01}$ and

$0<\delta_{j 19}<\delta_{j 14}<\delta_{j 15}<\delta_{j 16}<\delta_{j 17}<\delta_{j 19}<\delta_{j 19}<\delta_{j 20}<\delta_{j 21}<$

$\delta_{j 22}<\delta_{j 2 a}<\delta_{j 24}$

Implies c $>\mathrm{c}-\delta_{j 12}>\mathrm{c}-\delta_{j 11}>\mathrm{c}-\delta_{j 10}>\mathrm{c}-\delta_{j 09}>$

$\mathrm{c}-\delta_{j 08}>\mathrm{c}-\delta_{j 07}>\mathrm{c}-\delta_{j 06}>\mathrm{c}-\delta_{j 05}>\mathrm{c}-\delta_{j 04}>\mathrm{c}-\delta_{j 0 a}>\mathrm{c}-\delta_{j 02}>$

$\mathrm{C}-\delta_{j 01}$ x

$\mathrm{d}<\mathrm{d}+\delta_{j 1 \mathrm{a}}<\mathrm{d}+\delta_{j 14}<\mathrm{d}+\delta_{j 15}<\mathrm{d}+\delta_{j 16}<\mathrm{d}+\delta_{j 17}<\mathrm{d}+\delta_{j 18}<$ $\mathrm{d}+\delta_{j 19}<\mathrm{d}+\delta_{j 20}<\mathrm{d}+\delta_{j 21}<\mathrm{d}+\delta_{j 22}<\mathrm{d}+\delta_{j 2 a}<\mathrm{d}+\delta_{j 24}$

The graphical representation of generalized type-2 hexagonal fuzzy number defined in equation (8) is shown in the following Fig. 4.

Therefore the centroid of hexagonal fuzzy arc lengths is defined by using the proposed centroid of hexagonal fuzzy number (6) as follows:

$$
\begin{aligned}
& \mathrm{G}=(x(\tilde{\tilde{L}}), y(\tilde{\tilde{L}})) \\
& =\left(\frac { 1 } { \mathrm { g } 2 4 } \left\{162(c+d)+4\left(\delta_{j 14}+\delta_{j 24}-\delta_{j 01}-\delta_{j 11}\right)\right.\right. \\
& +6\left(\delta_{j 12}+\delta_{j 16}+\delta_{j 22}+\delta_{j 22}-\delta_{j 02}-\delta_{j 01}-\delta_{j 09}-\delta_{j 12}\right) \\
& 8\left(\delta_{j 18}+\delta_{j 20}-\delta_{j 05}-\delta_{j 07}\right)+9\left(\delta_{j 15}+\delta_{j 21}-\delta_{j 04}-\delta_{j 10}\right)+ \\
& \left.\left.12\left(\delta_{j 17}+\delta_{j 19}-\delta_{j 06}-\delta_{j 08}\right)\right\}, \frac{5 w_{d}}{18}\right)
\end{aligned}
$$

Therefore the ranking function for all possible arc lengths is defined as:

$$
\mathrm{R}(\tilde{\tilde{A}})=x(\widetilde{\widetilde{L}}) \alpha+(1-\alpha) y(\widetilde{\widetilde{L}})
$$

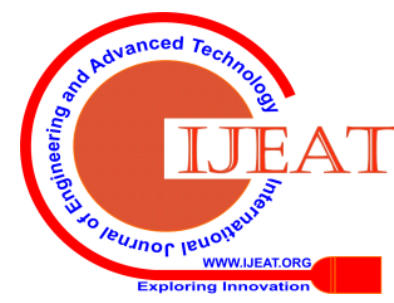




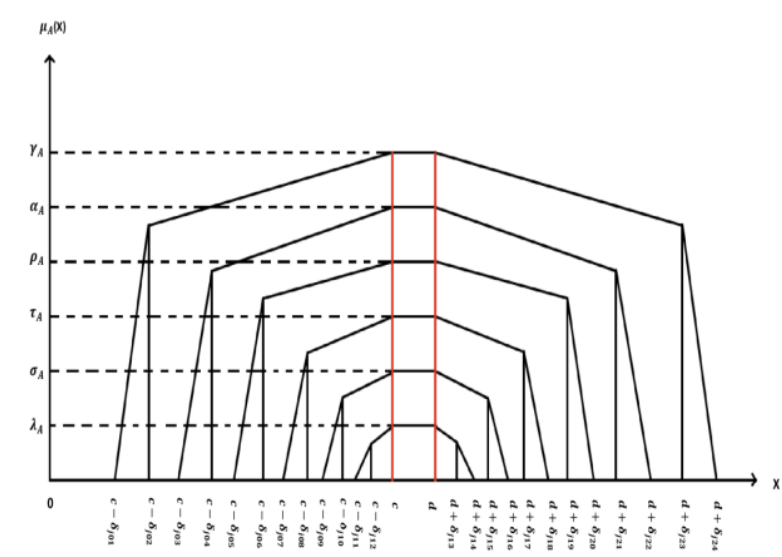

Fig 4. Generalized Type-2 Hexagonal Fuzzy Number

\section{Proposed ranking method of Generalized type-2 octagonal fuzzy number}

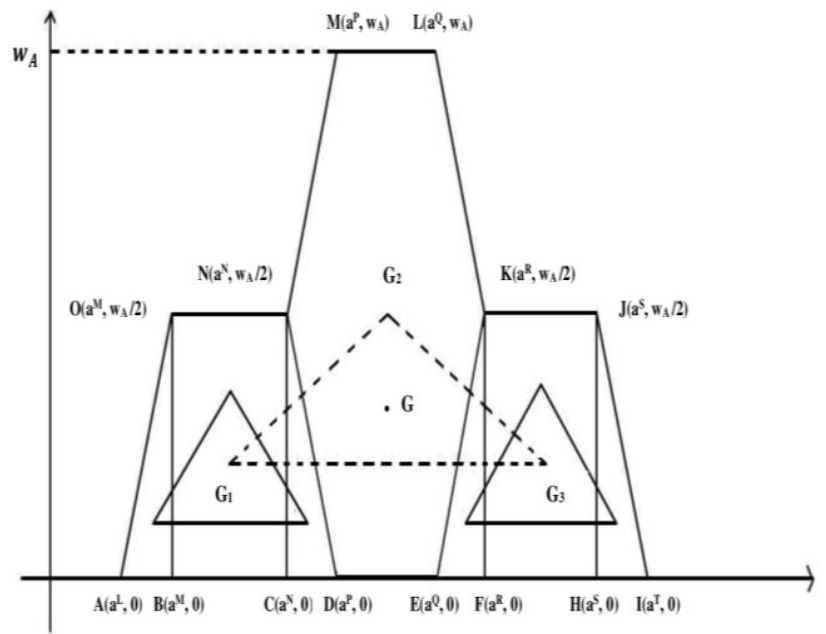

Fig 5. Centroid of Generalized Type-2 Octagonal Fuzzy Number

Centroid is the point of centre of gravity of any type of plane figure. The above plane figure is considered as the graphical representation of Generalized Type-2 Octagonal Fuzzy Number. In the plane Fig. 5, the $x$ co-ordinates $\mathrm{a}^{\mathrm{L}}, \mathrm{a}^{\mathrm{M}}, \mathrm{a}^{\mathrm{N}}, \mathrm{a}^{\mathrm{P}}, \mathrm{a}^{\mathrm{Q}}, \mathrm{a}^{\mathrm{R}}, \mathrm{a}^{\mathrm{S}}$ and $\mathrm{a}^{\mathrm{T}}$ of vertices are considered as centroid of centroids of each octagonal fuzzy quantities occurred in the Generalized Type-2 Octagonal Fuzzy Number. First, the octagon is divided into three plane figures AOND (trapezoid), DNMLKE (hexagon) and EKJI (trapezoid). The centroid of the centroids of the three plane figures is considered as the center of gravity point of generalized type- 2 octagonal fuzzy number to define its ranking function. The reason for selecting this point as a center point of gravity is that each Centroid point $\left(G_{1}\right.$ of trapezoid AOND, $G_{2}$ of hexagon DNMLKE, and $G_{3}$ of trapezoid EKJI) are center of gravity points of each individual plane figure. Therefore, the centroid of these gravity points might be a better equidistant point rather than centroid of octagon.

Consider Generalized Type-2 octagonal fuzzy number

$A=\left(\left(a_{1}^{L}, a_{2}^{L}, a_{2}^{L}, a_{4}^{L}, a_{5}^{L}, a_{6}^{L}, a_{7}^{L}, a_{9}^{L} ; \lambda_{A}\right)\right.$,

$\left(a_{1}^{M}, a_{2}^{M}, a_{2}^{M}, a_{4}^{M}, a_{5}^{M}, a_{6}^{M}, a_{7}^{M}, a_{g}^{M} ; \sigma_{A}\right)$,
$\left(a_{1}^{N}, a_{2}^{N}, a_{9}^{N}, a_{4}^{N}, a_{5}^{N}, a_{6}^{N}, a_{7}^{N}, a_{g}^{N} ; \tau_{A}\right)$,

$\left(a_{1}^{p}, a_{2}^{p}, a_{2}^{p}, a_{4}^{p}, a_{5}^{p}, a_{6}^{p}, a_{7}^{p}, a_{9}^{p} ; p_{A}\right)$,

$\left(a_{1}^{Q}, a_{2}^{Q}, a_{1}^{Q}, a_{4}^{Q}, a_{5}^{Q}, a_{6}^{Q}, a_{7}^{Q}, a_{8}^{Q} ; a_{A}\right)$,

$\left(a_{1}^{R}, a_{2}^{R}, a_{3}^{R}, a_{4}^{R}, a_{5}^{R}, a_{6}^{R}, a_{7}^{R}, a_{g}^{R} ; \beta_{A}\right)$,

$\left(a_{1}^{5}, a_{2}^{5}, a_{2}^{5}, a_{4,}^{5}, a_{5}^{5}, a_{6}^{5}, a_{7}^{5}, a_{9}^{5}, \gamma_{A}\right)$,

$\left.\left(a_{1}^{T}, a_{2}^{T}, a_{a}^{T}, a_{4}^{T}, a_{5}^{T}, a_{6}^{T}, a_{7}^{T}, a_{g}^{T} ; \delta_{A}\right) ; w_{A}\right)$

In figure 5 , the $\mathrm{x}$ co-ordinates $\mathrm{a}^{\mathrm{L}}, \mathrm{a}^{\mathrm{M}}, \mathrm{a}^{\mathrm{N}}, \mathrm{a}^{\mathrm{P}}, \mathrm{a}^{\mathrm{Q}}, \mathrm{a}^{\mathrm{R}}, \mathrm{a}^{\mathrm{S}}$ and $\mathrm{a}^{\mathrm{T}}$ are centroid of centroids of the octagonal fuzzy numbers

$\left(a_{1}^{L}, a_{2}^{L}, a_{9}^{L}, a_{4,}^{L}, a_{5}^{L}, a_{6}^{L}, a_{7}^{L}, a_{9}^{L}, \lambda_{A}\right)$,

$\left(a_{1}^{M}, a_{2}^{M}, a_{7}^{M}, a_{4}^{M}, a_{5}^{M}, a_{6}^{M}, a_{7}^{M}, a_{g}^{M}, \sigma_{A}\right)$,

$\left(a_{1}^{N}, a_{2}^{N}, a_{7}^{N}, a_{4}^{N}, a_{5}^{N}, a_{6}^{N}, a_{7}^{N}, a_{g}^{N} ; \tau_{A}\right)$,

$\left(a_{1}^{p}, a_{2}^{p}, a_{2}^{p}, a_{4}^{p}, a_{5}^{p}, a_{6}^{p} a_{7}^{p}, a_{9}^{p}, \rho_{A}\right)$,

$\left(a_{1}^{Q}, a_{2}^{Q}, a_{1}^{Q}, a_{4,}^{Q}, a_{5}^{Q}, a_{6}^{Q} a_{7}^{Q}, a_{8}^{Q}, a_{A}\right)$,

$\left(a_{1}^{R}, a_{2}^{R}, a_{1}^{R}, a_{4}^{R}, a_{5}^{R}, a_{6}^{R}, a_{7}^{R}, a_{2}^{R}, \beta_{A}\right)$,

$\left(a_{1}^{5}, a_{2}^{5}, a_{2}^{5}, a_{4,}^{5}, a_{5}^{5}, a_{6,}^{5}, a_{7}^{5}, a_{9}^{5} ; \gamma_{A}\right)$ and

$\left(a_{1}^{T}, a_{2}^{T}, a_{2}^{T}, a_{4}^{T}, a_{5}^{T}, a_{6}^{T}, a_{7}^{T}, a_{g}^{T} ; \delta_{A}\right)$ respectively.

For the sake of clarity, the centroid points of the three plane figures are presented as follows:

The centroid of AOND is

$G_{1}=\left(\frac{1}{972}\left\{4\left(a_{1}^{L}+a_{g}^{L}+a_{1}^{p}+a_{g}^{p}\right)+\right.\right.$

$14\left(a_{2}^{L}+a_{7}^{L}+a_{2}^{p}+a_{7}^{p}+a_{1}^{M}+a_{9}^{M}+a_{1}^{N}+a_{9}^{N}\right)$

$+20\left(a_{a}^{L}+a_{6}^{L}+a_{9}^{p}+a_{6}^{p}\right)+16\left(a_{4}^{L}+a_{5}^{L}+a_{4}^{p}+a_{5}^{p}\right)+$

$49\left(a_{2}^{N}+a_{7}^{N}+a_{2}^{M}+a_{7}^{M}\right)+56\left(a_{4}^{M}+a_{5}^{M}+a_{4}^{N}+a_{5}^{N}\right)+$

$\left.\left.70\left(a_{a}^{M}+a_{6}^{M}+a_{a}^{N}+a_{6}^{N}\right)\right\}, \frac{7 w_{A}}{36}\right)$

The centroid of DNMLKE is

$G_{2}=\left(\frac{1}{a 24}\left\{2\left(a_{1}^{N}+a_{g}^{N}+a_{1}^{R}+a_{g}^{R}\right)+4\left(a_{1}^{p}+a_{g}^{p}+a_{1}^{Q}+a_{g}^{Q}\right)\right.\right.$

$+7\left(a_{2}^{N}+a_{7}^{N}+a_{2}^{R}+a_{7}^{R}\right)+8\left(a_{4}^{N}+a_{5}^{N}+a_{4}^{R}+a_{5}^{R}\right)+$

$10\left(a_{a}^{N}+a_{6}^{N}+a_{a}^{R}+a_{6}^{R}\right)+14\left(a_{2}^{p}+a_{7}^{p}+a_{2}^{Q}+a_{7}^{Q}\right)+$

$\left.\left.16\left(a_{4}^{p}+a_{5}^{p}+a_{4}^{Q}+a_{5}^{Q}\right)+20\left(a_{3}^{p}+a_{6}^{p}+a_{a}^{Q}+a_{6}^{Q}\right)\right\}, \frac{a \omega_{A}}{6}\right)$

The centroid of EKJI is

$G_{a}=\left(\frac{1}{972}\left\{4\left(a_{1}^{Q}+a_{g}^{Q}+a_{1}^{T}+a_{g}^{T}\right)+\right.\right.$

$14\left(a_{2}^{Q}+a_{7}^{Q}+a_{2}^{T}+a_{7}^{T}+a_{1}^{R}+a_{g}^{R}+a_{1}^{5}+a_{g}^{5}\right)+$

$20\left(a_{3}^{Q}+a_{6}^{Q}+a_{a}^{T}+a_{6}^{T}\right)+16\left(a_{4}^{Q}+a_{5}^{Q}+a_{4}^{T}+a_{5}^{T}\right)+$

$49\left(a_{2}^{R}+a_{7}^{R}+a_{2}^{5}+a_{7}^{5}\right)+56\left(a_{4}^{R}+a_{5}^{R}+a_{4}^{5}+a_{5}^{5}\right)+$

$\left.\left.70\left(a_{a}^{R}+a_{6}^{R}+a_{a}^{5}+a_{6}^{5}\right)\right\}, \frac{7 \omega_{A}}{76}\right)$

Hence the centroid of $G_{1}, G_{2}$ and $G_{2}$ is

$\mathrm{G}=\left(x(\underset{\tilde{\widetilde{A}}}{)}, y(\underset{\tilde{A}}{\tilde{A}}))=\left(\frac{1}{2916}\left\{4\left(a_{1}^{L}+a_{g}^{L}+a_{1}^{T}+a_{g}^{T}\right)+\right.\right.\right.$

$14\left(a_{2}^{L}+a_{7}^{L}+a_{1}^{M}+a_{g}^{M}+a_{2}^{T}+a_{7}^{T}+a_{1}^{5}+a_{g}^{5}\right)+$

$16\left(a_{1}^{p}+a_{g}^{p}+a_{1}^{Q}+a_{8}^{Q}+a_{4}^{L}+a_{5}^{L}+a_{4}^{T}+a_{5}^{T}\right)+$

$20\left(a_{1}^{N}+a_{g}^{N}+a_{1}^{R}+a_{g}^{R}+a_{a}^{L}+a_{6}^{L}+a_{a}^{T}+a_{6}^{T}\right)+$

$49\left(a_{2}^{M}+a_{7}^{M}+a_{2}^{5}+a_{7}^{5}\right)+56\left(a_{2}^{p}+a_{7}^{p}+a_{2}^{Q}+a_{7}^{Q}+a_{4}^{M}+\right.$

$\left.a_{5}^{M}+a_{4}^{5}+a_{5}^{5}\right)+64\left(a_{4}^{p}+a_{5}^{p}+a_{4}^{Q}+a_{5}^{Q}\right)+70\left(a_{2}^{N}+a_{7}^{N}+\right.$

$\left.a_{2}^{R}+a_{7}^{R}+a_{2}^{M}+a_{6}^{M}+a_{2}^{5}+a_{6}^{5}\right)+80\left(a_{4}^{N}+a_{5}^{N}+a_{4}^{R}+\right.$

$\left.\left.a_{5}^{R}+a_{a}^{p}+a_{6}^{p}+a_{a}^{Q}+a_{6}^{Q}\right)+100\left(a_{a}^{R}+a_{6}^{R}+a_{a}^{N}+a_{6}^{N}\right)\right\}$,

$\left.\frac{B W_{A}}{27}\right)$

Then the ranking function of $\bar{A}$ is defined as

$\mathrm{R}(\tilde{A})=x(\stackrel{\widetilde{\tilde{A}}}{)} \alpha+(1-\alpha) y(\stackrel{\widetilde{\widetilde{A}}}{)}$

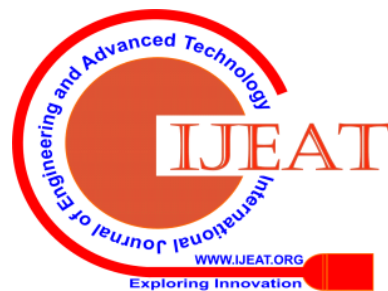


For all the possible paths, their corresponding path lengths $L_{j}, \mathrm{j}=1,2,3, \ldots \mathrm{n}$ to be fuzzified in an uncertain context, where $L_{j}=[\mathrm{d}$, e $]$ is considered as a generalized type-2 octagonal fuzzy number.

$\mathbb{L}_{\mathrm{g}}=[\mathrm{d}, \mathrm{e}]=$

$\left(\left(d-\delta_{j 22}, d-\delta_{j 2 a}, d-\delta_{j 24}, d_{,}, e+\delta_{j 25}, e+\delta_{j 26}, e+\delta_{j 27}\right)\right.$, $\left(\mathrm{d}-\delta_{j 19} \mathrm{~d}-\delta_{j 20}, \mathrm{~d}-\delta_{j 21}, \mathrm{~d}, \mathrm{e}, \mathrm{e}+\delta_{j 28}, \mathrm{e}+\delta_{j 29}, \mathrm{e}+\delta_{j 20}\right)$, $\left(\mathrm{d}-\delta_{j 16} \mathrm{~d}-\delta_{j 17}, \mathrm{~d}-\delta_{j 18}, \mathrm{~d}_{x} \mathrm{e}, \mathrm{e}+\delta_{j \mathrm{a1}}, \mathrm{e}+\delta_{j \mathrm{a} 2}, \mathrm{e}+\delta_{j \mathrm{a} a}\right)$, $\left(\mathrm{d}-\delta_{j 12}, \mathrm{~d}-\delta_{j 14}, \mathrm{~d}-\delta_{j 15}, \mathrm{~d}_{x} \mathrm{e}, \mathrm{e}+\delta_{j \mathrm{a},}, \mathrm{e}+\delta_{j \mathrm{a},}, \mathrm{e}+\delta_{j \mathrm{~g}}\right)_{x}$ $\left(\mathrm{d}-\delta_{j 10}, \mathrm{~d}-\delta_{j 11}, \mathrm{~d}-\delta_{j 12}, \mathrm{~d}, \mathrm{e}, \mathrm{e}+\delta_{j \mathrm{aj}}, \mathrm{e}+\delta_{j \mathrm{ag}}, \mathrm{e}+\delta_{\mathrm{jag}}\right)$, $\left(\mathrm{d}-\delta_{j 07}, \mathrm{~d}-\delta_{j 0 \mathrm{~g}}, \mathrm{~d}-\delta_{j 09,} \mathrm{~d}, \mathrm{e}, \mathrm{e}+\delta_{j 40}, \mathrm{e}+\delta_{j 41}, \mathrm{e}+\delta_{j 42}\right)$, $\left(\mathrm{d}-\delta_{j 04}, \mathrm{~d}-\delta_{j 05}, \mathrm{~d}-\delta_{j 06,} \mathrm{~d}_{x} \mathrm{e}, \mathrm{e}+\delta_{j 49} \mathrm{e}+\delta_{j 44}, \mathrm{e}+\delta_{j 45}\right)$, $\left(\mathrm{d}-\delta_{j 01}, \mathrm{~d}-\delta_{j 02}, \mathrm{~d}-\delta_{j 02}, \mathrm{~d}_{v} \mathrm{e}, \mathrm{e}+\delta_{j 46}, \mathrm{e}+\delta_{j 47}, \mathrm{e}+\delta_{j 48}\right)$

where

$0<\delta_{j 24}<\delta_{j 2 a}<\delta_{j 22}<\delta_{j 21}<\delta_{j 20}<\delta_{j 19}<\delta_{j 19}<\delta_{j 17}<$ $\delta_{j 16}<\delta_{j 15}<\delta_{j 14}<\delta_{j 1 a}<\delta_{j 12}<\delta_{j 11}<\delta_{j 10}<\delta_{j 09}<\delta_{j 09}<$ $\delta_{j 07}<\delta_{j 06}<\delta_{j 05}<\delta_{j 04}<\delta_{j 0 a}<\delta_{j 02}<\delta_{j 01}$ and $0<\delta_{j 25}<\delta_{j 26}<\delta_{j 27}<\delta_{j 29}<\delta_{j 29}<\delta_{j a 0}<\delta_{j a 1}<\delta_{j a 2}<$ $\delta_{j a a}<\delta_{j a 4}<\delta_{j a 5}<\delta_{j a 6}<\delta_{j a 7}<\delta_{j a 9}<\delta_{j a 9}<\delta_{j 40}<$ $\delta_{j 41}<\delta_{j 42}<\delta_{j 43}<\delta_{j 44}<\delta_{j 45}<\delta_{j 46}<\delta_{j 47}<\delta_{j 49}$ Implies

Therefore the centroid of octagonal fuzzy arc lengths is defined by using the proposed centroid of octagonal fuzzy number (11) as follows:

$\mathrm{G}=(x(\widetilde{\widetilde{L}}), y(\tilde{\widetilde{L}}))$

$=\left(\frac{1}{2916}\left\{1458(d+e)+4\left(\delta_{j 27}+\delta_{j 48}-\delta_{j 01}-\delta_{j 22}\right)\right.\right.$

$+14\left(\delta_{j 26}+\delta_{j \mathrm{j} 0}+\delta_{j 45}+\delta_{j 47}-\delta_{j 02}-\delta_{j 04}-\delta_{j 19}-\delta_{j 2 a}\right)$

$+16\left(\delta_{j 36}+\delta_{j a 9}-\delta_{j 10}-\delta_{j 13}\right)$

$+20\left(\delta_{j 25}+\delta_{j 19}+\delta_{j 42}+\delta_{j 46}-\delta_{j 01}-\delta_{j 07}-\delta_{j 16}-\delta_{j 24}\right)$

$+49\left(\delta_{j 29}+\delta_{j 44}-\delta_{j 05}-\delta_{j 20}\right)+56\left(\delta_{j 35}+\delta_{j 28}-\delta_{j 11}-\delta_{j 14}\right)+$

$70\left(\delta_{j 29}+\delta_{j \mathrm{j} 2}+\delta_{j 41}+\delta_{j 49}-\delta_{j 06}-\delta_{j 09}-\delta_{j 17}-\delta_{j 21}\right)$

$+80\left(\delta_{j a 4}+\delta_{j a 7}-\delta_{j 12}-\delta_{j 15}\right)+$

$\left.100\left(\delta_{j a 1}+\delta_{j 40}-\delta_{j 09}-\delta_{j 18}\right), \frac{B w_{A}}{27}\right)$

Therefore the ranking function for all possible arc lengths is defined as:

$$
\mathrm{R}(\tilde{A})=x(\stackrel{\widetilde{\tilde{L}}}{)} \alpha+(1-\alpha) y(\widetilde{\widetilde{L}})
$$

\section{Ranking Function}

Let $F(R)$ be the set of all generalized type-2 trapezoidal or hexagonal or octagonal fuzzy arc lengths. One feasible way for solving type-2 fuzzy critical path problem is based on the concept of comparison of type-2 fuzzy arc lengths by using ranking function. An effective approach for comparison of such fuzzy arc lengths is to define a ranking function $\mathbb{R}: \mathrm{F}(\mathrm{R}) \rightarrow \mathrm{R}$ which maps each type 2 fuzzy arc lengths into crisp one.

Using the proposed ranking functions defined on equations (5), (10) and (15), we define ranking between generalized type-2 trapezoidal or hexagonal or octagonal fuzzy arc lengths as follows:

$$
\begin{aligned}
& \text { (i) If } \mathbb{R}(\hat{A}) \geq \mathbb{R}(\hat{B}) \text {, then }(\hat{A}) \stackrel{\mathbb{R}}{\mathbb{R}}(\mathbb{B}) \\
& \text { (ii) If } \mathbb{R}(A) \leq \mathbb{R}(B) \text {, then }(A) \mathbb{R}(B) \\
& \text { (iii) If } \mathbb{R}(A)=\mathbb{R}(B) \text {, then }(A) \overline{\bar{R}}(B)
\end{aligned}
$$

$\mathrm{d}>\mathrm{d}-\delta_{j 24}>\mathrm{d}-\delta_{j 2 a}>\mathrm{d}-\delta_{j 22}>\mathrm{d}-\delta_{j 21}>\mathrm{d}-\delta_{j 20}>\mathrm{d}-\delta_{j 19}>$ $\mathrm{d}-\delta_{j 19}>\mathrm{d}-\delta_{j 17}>\mathrm{d}-\delta_{j 16}>\mathrm{d}-\delta_{j 15}>\mathrm{d}-\delta_{j 14}>\mathrm{d}-\delta_{j 13}>\mathrm{d}-\delta_{j 12}>$ $\mathrm{d}-\delta_{j 11}>\mathrm{d}-\delta_{j 10}>\mathrm{d}-\delta_{j 09}>\mathrm{d}-\delta_{j 08}>\mathrm{d}-\delta_{j 07}>\mathrm{d}-\delta_{j 06}>$

$\mathrm{d}-\delta_{j 05}>\mathrm{d}-\delta_{j 04}>\mathrm{d}-\delta_{j 03}>\mathrm{d}-\delta_{j 02}>\mathrm{d}-\delta_{j 01}$ and $\mathrm{e}<\mathrm{e}+\delta_{j 25}<\mathrm{e}+\delta_{j 26}<\mathrm{e}+\delta_{j 27}<\mathrm{e}+\delta_{j 2 \mathrm{~g}}<\mathrm{e}+\delta_{j 29}<\mathrm{e}+\delta_{j 10}<$ $\mathrm{e}+\delta_{j \mathrm{a} 1}<\mathrm{e}+\delta_{j \mathrm{ja}}<\mathrm{e}+\delta_{\mathrm{jaa}}<\mathrm{e}+\delta_{\mathrm{ja4}}<\mathrm{e}+\delta_{\mathrm{ja5}}<\mathrm{e}+\delta_{\mathrm{ja} 6}<\mathrm{e}+\delta_{\mathrm{ja} 7}<$ $\mathrm{e}+\delta_{j \mathrm{ag}}<\mathrm{e}+\delta_{j \mathrm{ag}}<\mathrm{e}+\delta_{j 40}<\mathrm{e}+\delta_{j 41}<\mathrm{e}+\delta_{j 42}<\mathrm{e}+\delta_{j 4 a}<$ $\mathrm{e}+\delta_{j 44}<\mathrm{e}+\delta_{j 45}<\mathrm{e}+\delta_{j 46}<\mathrm{e}+\delta_{j 47}<\mathrm{e}+\delta_{j 48}$

The graphical representation of generalized type-2 octagonal fuzzy number defined in equation (13) is shown in the following Fig. 6.

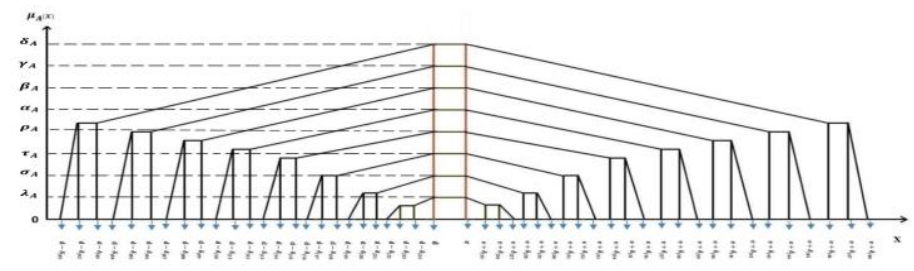

Fig.6. Generalized Type-2 Octagonal Fuzzy Number

\section{Algorithm}

STEP 1: In the project network, assign the fuzzy activity time of each activity for generalized type-2 trapezoidal fuzzy number

$A=\left(\left(a_{1}^{L}, a_{2}^{L}, a_{\mathrm{a}}^{L}, a_{4}^{L} ; \lambda_{A}\right),\left(a_{1}^{M}, a_{2}^{M}, a_{\mathrm{a}}^{M}, a_{4}^{M}, \sigma_{A}\right)\right.$, $\left.\left(a_{1}^{N}, a_{2}^{N}, a_{1}^{N}, a_{4}^{N}, \tau_{A}\right),\left(a_{1}^{P}, a_{2}^{p}, a_{1}^{p}, a_{4}^{p} ; p_{A}\right) ; \omega_{A}\right)$

or generalized type-2 hexagonal fuzzy number $A=$ $\left(\left(a_{1}^{L}, a_{2}^{L}, a_{\mathrm{a}}^{L}, a_{4}^{L}, a_{5}^{L}, a_{6}^{L}, \lambda_{A}\right),\left(a_{1}^{M}, a_{2}^{M}, a_{a}^{M}, a_{4}^{M}, a_{5}^{M}, a_{6}^{M} ; \sigma_{A}\right)\right.$, $\left(a_{1}^{N}, a_{2}^{N}, a_{1}^{N}, a_{4}^{N}, a_{5}^{N}, a_{6}^{N} ; \tau_{A}\right),\left(a_{1}^{p}, a_{2}^{p}, a_{1}^{p}, a_{4}^{p}, a_{5}^{p}, a_{6}^{p} ; p_{A}\right)$, $\left.\left(a_{1}^{Q}, a_{2}^{Q}, a_{3}^{Q}, a_{4}^{Q}, a_{5}^{Q}, a_{6}^{Q} ; a_{A}\right),\left(a_{1}^{R}, a_{2}^{R}, a_{3}^{R}, a_{4}^{R}, a_{5}^{R}, a_{6}^{R} ; \gamma_{A}\right) ; w_{A}\right)$ or generalized type-2 octagonal fuzzy number

$A=\left(\left(a_{1}^{L}, a_{2}^{L}, a_{5}^{L}, a_{4}^{L}, a_{5}^{L}, a_{6}^{L}, a_{7}^{L}, a_{9}^{L}, \lambda_{A}\right)\right.$,

$\left(a_{1}^{M}, a_{2}^{M}, a_{\mathrm{a}}^{M}, a_{4}^{M}, a_{5}^{M}, a_{6}^{M}, a_{7}^{M}, a_{g}^{M} ; \sigma_{A}\right)$,

$\left(a_{1}^{N}, a_{2}^{N}, a_{7}^{N}, a_{4}^{N}, a_{5}^{N}, a_{6}^{N}, a_{7}^{N}, a_{8}^{N}, \tau_{A}\right)$,

$\left(a_{1}^{p}, a_{2}^{p}, a_{1}^{p}, a_{4}^{p}, a_{5}^{p}, a_{6}^{p}, a_{7}^{p}, a_{9}^{p} ; p_{A}\right)$,

$\left(a_{1}^{Q}, a_{2}^{Q}, a_{1}^{Q}, a_{4}^{Q}, a_{5}^{Q}, a_{6}^{Q}, a_{7}^{Q}, a_{8}^{Q}, a_{A}\right)$,

$\left(a_{1}^{R}, a_{2}^{R}, a_{1}^{R}, a_{4}^{R}, a_{5}^{R}, a_{6}^{R}, a_{7}^{R}, a_{9}^{R} ; \beta_{A}\right)$,

$\left(a_{1}^{5}, a_{2}^{5}, a_{2}^{5}, a_{4,}^{5}, a_{5}^{5}, a_{6,}^{5}, a_{7}^{5}, a_{9}^{5}, \gamma_{A}\right)$,

$\left.\left(a_{1}^{T}, a_{2}^{T}, a_{9}^{T}, a_{4}^{T}, a_{5}^{T}, a_{6}^{T}, a_{7}^{T}, a_{9}^{T} ; \delta_{A}\right) ; \omega_{A}\right)$

STEP 2 : Calculate Earliest fuzzy starting time $E_{m}^{\mathrm{s}}$ 's with the earliest occurrence time of zero for the initial event and Earliest fuzzy finishing time $E_{\mathrm{m}}^{f}$ 's using

$$
E_{m}^{\mathbb{s}}=\max _{n \in p(m)}\left\{E_{n}^{s} \oplus f_{n}^{*}\right\} \text { and } E_{m}^{f}=E_{m}^{\mathbb{f}} \oplus F_{n}^{s}
$$

STEP 3 : Calculate Latest fuzzy finishing time $\mathcal{L}_{\mathrm{m}}^{\tilde{f}}$ 's and Latest fuzzy starting time $\tilde{\mathbb{L}_{m}^{f}}$ 's using $\widetilde{L}_{\mathrm{m}}^{\mathcal{f}}=$ $\min _{n \in p(m)}\left\{\widetilde{L_{n}^{f}} \theta \tilde{t}_{n}^{s}\right\}$ and $\tilde{L_{m}^{*}}=\widetilde{L_{m}^{f}} \theta f_{n}^{s}$

STEP 4 : Calculate Total float fuzzy time $\mathbb{T}_{\mathrm{m}}^{\mathbf{F}}$, s for each activity $(\mathrm{m}, \mathrm{n})$ using

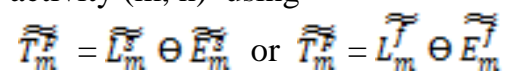


STEP 5 : Find all the possible paths and compute the total float fuzzy time of each path.

STEP 6 : Transform the total float fuzzy time of each path into standardized total float fuzzy time

( For example, if $A=\left(\left(a_{1}^{L}, a_{2}^{L}, a_{1,}^{L}, a_{4}^{L}\right)\right.$,

$\left.\left(a_{1}^{M}, a_{2}^{M}, a_{a}^{M}, a_{4}^{M}\right),\left(a_{1}^{N}, a_{2}^{N}, a_{a}^{N}, a_{4}^{N}\right),\left(a_{1}^{p}, a_{2}^{p}, a_{9}^{p}, a_{4}^{p}\right)\right)$ is the total float fuzzy time of the path, then its standardized total float fuzzy time is

$\left(\left(\frac{a_{1}^{L}}{K}, \frac{a_{2}^{L}}{K}, \frac{a_{3}^{L}}{K}, \frac{a_{4}^{L}}{K}\right),\left(\frac{a_{1}^{M}}{K}, \frac{a_{2}^{M}}{K}, \frac{a_{3}^{M}}{K}, \frac{a_{4}^{M}}{K}\right),\left(\frac{a_{1}^{N}}{K}, \frac{a_{2}^{N}}{K}, \frac{a_{2}^{N}}{K}, \frac{a_{4}^{N}}{K}\right),\left(\frac{a_{1}^{F}}{K}, \frac{a_{2}^{F}}{K}, \frac{a_{2}^{F}}{K}, \frac{a_{4}^{F}}{K}\right)\right)$ where $K=\max \left\|a_{\mathrm{f}}^{j}\right\|,\left\|a_{\mathrm{f}}^{j}\right\|$ denotes the upper bound of $\mid \alpha_{\mathrm{f}}^{\tilde{f}} \|, 1 \leq \mathrm{i}, \mathrm{j} \leq 4$

STEP 7 : Rank the standardized total float fuzzy time of each path using (5) (or (10) or (15)) and find the critical path.

\section{NUMERICAL EXAMPLE}

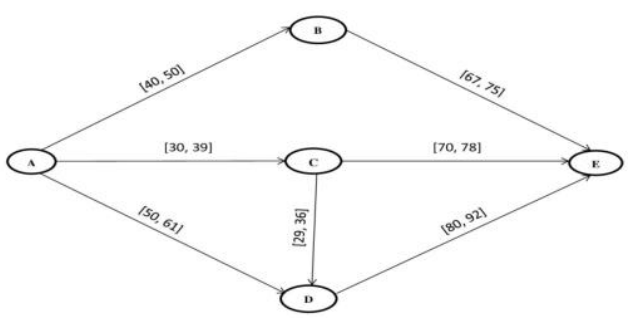

Fig 7. Network diagram for critical path

A salesman travels from a city A to the city E. The travelling time in hours between two cities may vary according to the distances which are given in the Fig. 7. Which way would take a long time for him to reach the destiny E?

\section{A. Case 1: Solution of critical path problem based on Generalized type-2 trapezoidal fuzzy number}

As per the proposed algorithm, the calculations have been carried out based on Generalized Type-2 Trapezoidal Fuzzy Numbers using the proposed ranking function (5). The results of the calculations are presented in the following tables.

Table - I: Generalized Type-2 Trapezoidal Total Float Fuzzy Time of each activity

\begin{tabular}{|c|c|c|}
\hline Activity & $\begin{array}{c}\text { Generalized Type - 2 } \\
\text { Trapezoidal Fuzzy Activity } \\
\text { Time }\end{array}$ & $\begin{array}{c}\text { Generalized Type - 2 } \\
\text { Trapezoidal Total Float } \\
\text { Fuzzy Time }\end{array}$ \\
\hline \multirow{2}{*}{ A - B } & $\begin{array}{c}((37,40,50,53),(28,40,50,64), \\
(25,40,50,67),(16,40,50,78))\end{array}$ & $\begin{array}{c}((0,14,60,79),(0,14,60,131), \\
(0,14,60,147),(0,14,60,197))\end{array}$ \\
\hline \multirow{2}{*}{ A - C } & $\begin{array}{c}((25,30,39,42),(16,30,39,53), \\
(13,30,39,56),(4,30,39,67))\end{array}$ & $\begin{array}{c}((0,0,28,51),(0,0,28,111), \\
(0,0,28,130),(0,0,28,189))\end{array}$ \\
\hline \multirow{2}{*}{ A - D } & $\begin{array}{c}((46,50,61,64),(37,50,61,75), \\
(34,50,61,78),(25,50,61,89))\end{array}$ & $\begin{array}{c}((0,0,37,55),(0,0,37,106), \\
(0,0,37,122),(0,0,37,172))\end{array}$ \\
\hline \multirow{2}{*}{ C - D } & $\begin{array}{c}((25,29,36,39),(16,29,36,50), \\
(13,29,36,53),(4,29,36,64))\end{array}$ & $\begin{array}{c}((0,0,28,51),(0,0,28,111), \\
(0,0,28,130),(0,0,28,189))\end{array}$ \\
\hline \multirow{2}{*}{ B - E } & $\begin{array}{c}((60,67,75,78),(50,67,75,89), \\
(47,67,75,93),(38,67,75,104))\end{array}$ & $\begin{array}{c}((0,14,60,79),(0,14,60,131), \\
(0,14,60,147),(0,14,60,197))\end{array}$ \\
\hline \multirow{2}{*}{ C - E } & $\begin{array}{c}((66,70,78,81),(57,70,78,92), \\
(54,70,78,95),(45,70,78,106))\end{array}$ & $\begin{array}{c}((2,22,67,85),(0,22,67,136), \\
(0,22,67,152),(0,22,67,202))\end{array}$ \\
\hline \multirow{2}{*}{ D - E } & $\begin{array}{c}((75,80,92,95),(66,80,92,106), \\
(63,80,92,110),(54,80,92,120))\end{array}$ & $\begin{array}{c}((0,0,28,51),(0,0,28,111), \\
(0,0,28,130),(0,0,28,189))\end{array}$ \\
\hline
\end{tabular}

Table - II: Generalized Type-2 Trapezoidal Standardized total float fuzzy time for each path

\begin{tabular}{|c|c|c|}
\hline Path & $\begin{array}{c}\text { Generalized Type-2 } \\
\text { Trapezoidal Total }\end{array}$ & $\begin{array}{c}\text { Generalized Type-2 } \\
\text { Trapezoidal Standardized }\end{array}$ \\
\hline
\end{tabular}

\begin{tabular}{|c|c|c|}
\hline & Float Fuzzy Time & Total Float Fuzzy Time \\
\hline & $((0,28,120,158)$, & $((0,0.07,0.3,0.4)$, \\
A-B - E & $(0,28,120,262)$, & $(0,0.07,0.3,0.66)$, \\
& $(0,28,120,294)$, & $(0,0.07,0.3,0.75)$, \\
& $(0,28,120,394))$ & $(0,0.07,0.3,1))$ \\
\hline & $((0,22,95,136)$, & $((0,0.06,0.24,0.35)$, \\
A-C-E & $(0,22,95,247)$, & $(0,0.06,0.24,0.63)$, \\
& $(0,22,95,282)$, & $(0,0.06,0.24,0.72)$, \\
& $(0,22,95,391))$ & $(0,0.06,0.24,1))$ \\
\hline & $((0,0,84,153)$, & $((0,0,0.15,0.27)$, \\
A-C-D-E & $(0,0,84,333)$, & $(0,0,0.15,0.59)$, \\
& $(0,0,84,390)$, & $(0,0,0.15,0.69)$, \\
& $(0,0,84,567))$ & $(0,0,0.15,1))$ \\
\hline & $((0,0,65,106)$, & $((0,0,0.18,0.29)$, \\
A-D - E & $(0,0,65,217)$, & $(0,0,0.18,0.6)$, \\
& $(0,0,65,252)$, & $(0,0,0.18,0.7)$, \\
& $(0,0,65,361))$ & $(0,0,0.18,1))$ \\
\hline
\end{tabular}

By using (5), we get

For $\alpha=1$,

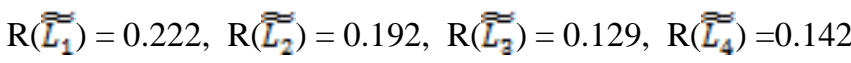
For $\alpha=0.5$,

$\mathrm{R}\left(\tilde{L_{1}}\right)=0.305, \mathrm{R}\left(\tilde{L}_{2}^{E}\right)=0.290, \mathrm{R}\left(\overline{\mathscr{L}}_{\mathrm{a}}\right)=0.256, \mathrm{R}\left(\tilde{L}_{4}\right)=0.265$ For $\alpha=0$,

$\mathrm{R}\left(\widetilde{L}_{1}\right)=\mathrm{R}\left(\widetilde{L}_{2}\right)=\mathrm{R}\left(\widetilde{L}_{\mathfrak{g}}\right)=\mathrm{R}\left(\widetilde{L}_{4}\right)=0.389$

Here minimum ranks are $0.129,0.256$ and 0.389 for $\alpha=1$, $\alpha=0.5$ and $\alpha=0$ respectively.

So the critical path is $\mathrm{A}-\mathrm{C}-\mathrm{D}-\mathrm{E}$

B. Case 2: Solution of critical path problem based on Generalized type-2 hexagonal fuzzy number

As per the proposed algorithm, the calculations have been carried out based on

Generalized Type-2 Hexagonal Fuzzy Numbers using the proposed ranking function (10). The results of the calculations are presented in the following tables.

Table - III: Generalized Type-2 Hexagonal Total Float Fuzzy Time of each activity

\begin{tabular}{|c|c|c|}
\hline \multirow{3}{*}{ Activity } & $\begin{array}{c}\text { Generalized Type }-2 \\
\text { Hexagonal Fuzzy } \\
\text { Activity Time }\end{array}$ & $\begin{array}{c}\text { Generalized Type }-2 \\
\text { Hexagonal Total } \\
\text { Float Fuzzy Time }\end{array}$ \\
\hline \multirow{5}{*}{ A - B } & $((37,39,40,50,51,53)$, & $((0,4,14,60,68,79)$, \\
& $(31,33,40,50,58,60)$, & $(0,0,14,60,102,112)$, \\
& $(28,30,40,50,61,64)$, & $(0,0,14,60,117,131)$, \\
& $(25,27,40,50,65,67)$, & $(0,0,14,60,136,147)$, \\
& $(22,24,40,50,68,71)$, & $(0,0,14,60,152,164)$, \\
& $(16,18,40,50,75,78))$ & $(0,0,14,60,185,197))$ \\
\hline & $((25,27,30,39,40,42)$, & $((0,0,0,28,39,51)$, \\
& $(19,21,30,39,47,49)$, & $(0,0,0,28,78,90)$, \\
& $(16,18,30,39,50,53)$, & $(0,0,0,28,96,111)$, \\
& $(13,15,30,39,54,56)$, & $(0,0,0,28,117,130)$, \\
& $(10,12,30,39,57,60)$, & $(0,0,0,28,136,150)$, \\
& $(4,6,30,39,64,67))$ & $(0,0,0,28,175,189))$ \\
\hline \multirow{5}{*}{ A - D } & $((46,48,50,61,62,64)$, & $((0,0,0,37,45,55)$, \\
& $(40,42,50,61,69,71)$, & $(0,0,0,37,78,88)$, \\
& $(37,39,50,61,72,75)$, & $(0,0,0,37,93,106)$, \\
& $(34,36,50,61,76,78)$, & $(0,0,0,37,111,122)$, \\
& $(31,33,50,61,79,82)$, & $(0,0,0,37,127,139)$, \\
& $(25,27,50,61,86,89))$ & $(0,0,0,37,160,172))$ \\
\hline
\end{tabular}

Published By:

Blue Eyes Intelligence Engineering \& Sciences Publication 


\begin{tabular}{|c|c|c|}
\hline & $((25,27,29,36,37,39)$, & $((0,0,0,28,39,51)$, \\
& $(19,21,29,36,44,46)$, & $(0,0,0,28,78,90)$, \\
C-D & $(16,18,29,36,47,50)$, & $(0,0,0,28,96,111)$, \\
& $(13,15,29,36,51,53)$, & $(0,0,0,28,117,130)$, \\
& $(10,12,29,36,54,57)$, & $(0,0,0,28,136,150)$, \\
& $(4,6,29,36,61,64))$ & $(0,0,0,28,175,189))$ \\
\hline \multirow{5}{*}{ B-E E } & $((60,63,67,75,76,78)$, & $((0,4,14,60,68,79)$, \\
& $(54,56,67,75,83,86)$, & $(0,0,14,60,102,112)$, \\
& $(50,53,67,75,87,89)$, & $(0,0,14,60,117,131)$, \\
& $(47,49,67,75,91,93)$, & $(0,0,14,60,136,147)$, \\
& $(44,46,67,75,94,97)$, & $(0,0,14,60,152,164)$, \\
& $(38,40,67,75,101,104))$ & $(0,0,14,60,185,197))$ \\
\hline \multirow{5}{*}{$-\mathrm{E}$} & $((66,68,70,78,79,81)$, & $((2,12,22,67,75,85)$, \\
& $(60,62,70,78,86,88)$, & $(0,0,22,67,108,118)$, \\
& $(57,59,70,78,89,92)$, & $(0,0,22,67,123,136)$, \\
& $(54,56,70,78,93,95)$, & $(0,0,22,67,141,152)$, \\
& $(51,53,70,78,96,99)$, & $(0,0,22,67,157,169)$, \\
& $(45,47,70,78,103,106))$ & $(0,0,22,67,190,202))$ \\
\hline & $((75,77,80,92,93,95)$, & $((0,0,0,28,39,51)$, \\
& $(69,71,80,92,100,102)$, & $(0,0,0,28,78,90)$, \\
D-E & $(66,68,80,92,103,106)$, & $(0,0,0,28,96,111)$, \\
& $(63,65,80,92,107,110)$, & $(0,0,0,28,117,130)$, \\
& $(60,62,80,92,111,113)$, & $(0,0,0,28,136,150)$, \\
& $(54,56,80,92,118,120))$ & $(0,0,0,28,175,189))$ \\
\hline
\end{tabular}

Table - IV: Generalized Type-2 Hexagonal Standardized total float fuzzy time for each path

\begin{tabular}{|c|c|c|}
\hline Path & $\begin{array}{c}\text { Generalized Type-2 } \\
\text { Hexagonal Total Float } \\
\text { Fuzzy Time }\end{array}$ & $\begin{array}{c}\text { Generalized Type-2 } \\
\text { Hexagonal Standardized } \\
\text { Total Float Fuzzy Time }\end{array}$ \\
\hline \multirow{5}{*}{ A-B-E } & $((0,8,28,120,136,158)$, & $((0,0.02,0.07,0.3,0.35,0.4)$, \\
& $(0,0,28,120,204,224)$, & $(0,0,0.07,0.3,0.52,0.57)$, \\
& $(0,0,28,120,234,262)$, & $(0,0,0.07,0.3,0.59,0.66)$, \\
& $(0,0,28,120,272,294)$, & $(0,0,0.07,0.3,0.69,0.75)$, \\
& $(0,0,28,120,304,328)$, & $(0,0,0.07,0.3,0.77,0.83)$, \\
& $(0,0,28,120,370,394))$ & $(0,0,0.07,0.3,0.94,1))$ \\
\hline \multirow{5}{*}{ A-C-E } & $((2,12,22,95,114,136)$, & $((0,0.03,0.06,0.24,0.29,0.35)$, \\
& $(0,0,22,95,186,208)$, & $(0,0,0.06,0.24,0.48,0.53)$, \\
& $(0,0,22,95,219,247)$, & $(0,0,0.06,0.24,0.56,0.63)$, \\
& $(0,0,22,95,258,282)$, & $(0,0,0.06,0.24,0.66,0.72)$, \\
& $(0,0,22,95,293,319)$, & $(0,0,0.06,0.24,0.75,0.82)$, \\
& $(0,0,22,95,365,391))$ & $(0,0,0.06,0.24,0.93,1))$ \\
\hline & $((0,0,0,84,117,153)$, & $((0,0,0,0.15,0.21,0.27)$, \\
& $(0,0,0,84,234,270)$, & $(0,0,0,0.15,0.41,0.48)$, \\
& $(0,0,0,84,288,333)$, & $(0,0,0,0.15,0.51,0.59)$, \\
& $(0,0,0,84,351,390)$, & $(0,0,0,0.15,0.62,0.69)$, \\
& $(0,0,0,84,408,450)$, & $(0,0,0,0.15,0.72,0.79)$, \\
& $(0,0,0,84,525,567))$ & $(0,0,0,0.15,0.93,1))$ \\
\hline A-D-E & $((0,0,0,65,84,106)$, & $((0,0,0,0.18,0.23,0.29)$, \\
& $(0,0,0,65,156,178)$, & $(0,0,0,0.18,0.43,0.49)$, \\
& $(0,0,0,65,189,217)$, & $(0,0,0,0.18,0.52,0.6)$, \\
& $(0,0,0,65,228,252)$, & $(0,0,0,0.18,0.63,0.7)$, \\
& $(0,0,0,65,263,289)$, & $(0,0,0,0.18,0.73,0.8)$, \\
\hline
\end{tabular}

By using (10), we get

For $\alpha=1$,

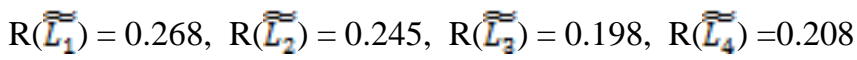
For $\alpha=0.5$,

$\mathrm{R}\left(\widetilde{\mathscr{L}_{1}}\right)=0.273, \mathrm{R}\left(\tilde{L}_{2}\right)=0.261, \mathrm{R}\left(\widetilde{\mathscr{L}_{3}}\right)=0.238, \mathrm{R}\left(\widetilde{\mathscr{L}_{4}}\right)=0.243$ For $\alpha=0$,

$\mathrm{R}\left(\tilde{\tilde{L}_{1}}\right)=\mathrm{R}\left(\tilde{\tilde{L}_{2}}\right)=\mathrm{R}\left(\widetilde{\mathscr{L}_{2}}\right)=\mathrm{R}\left(\tilde{\tilde{L}_{4}}\right)=0.278$

Here minimum ranks are $0.198,0.238$ and 0.278 for $\alpha=1$, $\alpha=0.5$ and $\alpha=0$ respectively.

So the critical path is $\mathrm{A}-\mathrm{C}-\mathrm{D}-\mathrm{E}$.

\section{Case 3: Solution of critical path problem based on Generalized type-2 octagonal fuzzy number}

As per the proposed algorithm, the calculations have been carried out based on Generalized Type-2 Octagonal Fuzzy Numbers using the proposed ranking function (15). The results of the calculations are presented in the following tables.

Table - V: Generalized Type-2 Octagonal Total Float Fuzzy Time of each activity

\begin{tabular}{|c|c|c|}
\hline Activity & $\begin{array}{c}\text { Generalized Type - } 2 \\
\text { Octagonal } \\
\text { Fuzzy Activity Time }\end{array}$ & $\begin{array}{c}\text { Generalized Type - } 2 \\
\text { Octagonal } \\
\text { Total Float Fuzzy Time }\end{array}$ \\
\hline$A-B$ & $\begin{array}{c}((37,38,39,40,50,51,52,53), \\
(34,35,36,40,50,54,55,57), \\
(31,32,33,40,50,58,59,60), \\
(28,29,30,40,50,61,62,64), \\
(25,26,27,40,50,65,66,67), \\
(22,23,24,40,50,68,69,71), \\
(19,20,21,40,50,72,73,74), \\
(16,17,18,40,50,75,76,78))\end{array}$ & $\begin{array}{c}((0,0,4,14,60,68,73,79), \\
(0,0,0,14,60,84,89,97), \\
(0,0,0,14,60,102,107,112), \\
(0,0,0,14,60,117,123,131), \\
(0,0,0,14,60,136,141,147), \\
(0,0,0,14,60,152,157,164), \\
(0,0,0,14,60,169,174,180), \\
(0,0,0,14,60,185,190,197))\end{array}$ \\
\hline$A-C$ & $\begin{array}{c}((25,26,27,30,39,40,41,42), \\
(22,23,24,30,39,43,44,46), \\
(19,20,21,30,39,47,48,49) \\
(16,17,18,30,39,50,51,53) \\
(13,14,15,30,39,54,55,56) \\
(10,11,12,30,39,57,58,60) \\
(7,8,9,30,39,61,62,63) \\
(4,5,6,30,39,64,65,67))\end{array}$ & $\begin{array}{c}((0,0,0,0,28,39,45,51), \\
(0,0,0,0,28,57,63,72), \\
(0,0,0,0,28,78,84,90), \\
(0,0,0,0,28,96,102,111), \\
(0,0,0,0,28,117,123,130), \\
(0,0,0,0,28,136,142,150), \\
(0,0,0,0,28,156,162,169), \\
(0,0,0,0,28,175,181,189))\end{array}$ \\
\hline$A-D$ & $\begin{array}{l}((46,47,48,50,61,62,63,64), \\
(43,44,45,50,61,65,66,68), \\
(40,41,42,50,61,69,70,71), \\
(37,38,39,50,61,72,73,75), \\
(34,35,36,50,61,76,77,78), \\
(31,32,33,50,61,79,80,82), \\
(28,29,30,50,61,83,84,85), \\
(25,26,27,50,61,86,87,89))\end{array}$ & $\begin{array}{l}((0,0,0,0,37,45,50,55), \\
(0,0,0,0,37,60,65,73), \\
(0,0,0,0,37,78,83,88), \\
(0,0,0,0,37,93,98,106), \\
(0,0,0,0,37,111,116,122), \\
(0,0,0,0,37,127,132,139), \\
(0,0,0,0,37,144,149,155), \\
(0,0,0,0,37,160,165,172))\end{array}$ \\
\hline$C-D$ & $\begin{array}{l}((25,26,27,29,36,37,38,39), \\
(22,23,24,29,36,40,41,43), \\
(19,20,21,29,36,44,45,46), \\
(16,17,18,29,36,47,48,50) \\
(13,14,15,29,36,51,52,53), \\
(10,11,12,29,36,54,55,57), \\
(7,8,9,29,36,58,59,60), \\
(4,5,6,29,36,61,62,64))\end{array}$ & $\begin{array}{l}((0,0,0,0,28,39,45,51), \\
(0,0,0,0,28,57,63,72), \\
(0,0,0,0,28,78,84,90), \\
(0,0,0,0,28,96,102,111), \\
(0,0,0,0,28,117,123,130), \\
(0,0,0,0,28,136,142,150), \\
(0,0,0,0,28,156,162,169), \\
(0,0,0,0,28,175,181,189))\end{array}$ \\
\hline $\mathrm{B}-\mathrm{E}$ & $\begin{array}{l}((60,62,63,67,75,76,77,78), \\
(57,58,59,67,75,80,81,82), \\
(54,55,56,67,75,83,84,86), \\
(50,51,53,67,75,87,88,89), \\
(47,48,49,67,75,91,92,93), \\
(44,45,46,67,75,94,95,97), \\
(41,42,43,67,75,98,99,100), \\
(38,39,40,67,75,101,102,104))\end{array}$ & $\begin{array}{l}((0,0,4,14,60,68,73,79), \\
(0,0,0,14,60,84,89,97), \\
(0,0,0,14,60,102,107,112), \\
(0,0,0,14,60,117,123,131), \\
(0,0,0,14,60,136,141,147), \\
(0,0,0,14,60,152,157,164), \\
(0,0,0,14,60,169,174,180), \\
(0,0,0,14,60,185,190,197))\end{array}$ \\
\hline$C-E$ & $\begin{array}{l}((66,67,68,70,78,79,80,81), \\
(63,64,65,70,78,82,83,85), \\
(60,61,62,70,78,86,87,88), \\
(57,58,59,70,78,89,90,92), \\
(54,55,56,70,78,93,94,95), \\
(51,52,53,70,78,96,97,99), \\
(48,49,50,70,78,100,101,102), \\
(45,46,47,70,78,103,104,106))\end{array}$ & $\begin{array}{l}((2,7,12,22,67,75,80,85), \\
(0,0,0,22,67,90,95,103), \\
(0,0,0,22,67,108,113,118), \\
(0,0,0,22,67,123,128,136), \\
(0,0,0,22,67,141,146,152), \\
(0,0,0,22,67,157,162,169), \\
(0,0,0,22,67,174,179,185), \\
(0,0,0,22,67,190,195,202))\end{array}$ \\
\hline$D-E$ & $\begin{array}{l}((75,76,77,80,92,93,94,95), \\
(72,73,74,80,92,96,97,99), \\
(69,70,71,80,92,100,101,102), \\
(66,67,68,80,92,103,104,106), \\
(63,64,65,80,92,107,108,110), \\
(60,61,62,80,92,111,112,113), \\
(57,58,59,80,92,114,115,117), \\
(54,55,56,80,92,118,119,120))\end{array}$ & $\begin{array}{l}((0,0,0,0,28,39,45,51), \\
(0,0,0,0,28,57,63,72), \\
(0,0,0,0,28,78,84,90), \\
(0,0,0,0,28,96,102,111), \\
(0,0,0,0,28,117,123,130), \\
(0,0,0,0,28,136,142,150), \\
(0,0,0,0,28,156,162,169), \\
(0,0,0,0,28,175,181,189))\end{array}$ \\
\hline
\end{tabular}

Table - VI: Generalized Type-2 Octagonal Standardized total float fuzzy time for each path

\begin{tabular}{|c|c|c|}
\hline Path & $\begin{array}{c}\text { Generalized Type-2 } \\
\text { Octagonal Total } \\
\text { Float Fuzzy Time }\end{array}$ & $\begin{array}{c}\text { Generalized Type-2 } \\
\text { Octagonal Standardized } \\
\text { Total } \\
\text { Float Fuzzy Time }\end{array}$ \\
\hline
\end{tabular}




\begin{tabular}{|c|c|c|}
\hline & $((0,0,8,28,120,136,146,158)$, & $((0,0, .02, .07, .3, .35, .37, .4)$, \\
& $(0,0,0,28,120,168,178,194)$, & $(0,0,0, .07, .3, .43, .45, .49)$, \\
& $(0,0,0,28,120,204,214,224)$, & $(0,0,0, .07, .3, .52, .54, .57)$, \\
A-B-E & $(0,0,0,28,120,234,246,262)$, & $(0,0,0, .07, .3, .59, .62, .66)$, \\
& $(0,0,0,28,120,272,282,294)$, & $(0,0,0, .07, .3, .69, .72, .75)$, \\
& $(0,0,0,28,120,304,314,328)$, & $(0,0,0, .07, .3, .77, .8, .83)$, \\
& $(0,0,0,28,120,338,348,360)$, & $(0,0,0, .07, .3, .86, .88, .91)$, \\
& $(0,0,0,28,120,370,380,394))$ & $(0,0,0, .07, .3, .94, .96,1))$ \\
\hline & $((2,7,12,22,95,114,125,136)$, & $((0, .02, .03, .06, .24, .29, .32, .35)$, \\
& $(0,0,0,22,95,147,158,175)$, & $(0,0,0, .06, .24, .38, .4, .45)$, \\
& $(0,0,0,22,95,186,197,208)$, & $(0,0,0, .06, .24, .48, .5, .53)$, \\
A-C-E & $(0,0,0,22,95,219,230,247)$, & $(0,0,0, .06, .24, .56, .59, .63)$, \\
& $(0,0,0,22,95,258,269,282)$, & $(0,0,0, .06, .24, .66, .69, .72)$, \\
& $(0,0,0,22,95,293,304,319)$, & $(0,0,0, .06, .24, .75, .78, .82)$, \\
& $(0,0,0,22,95,330,341,354)$, & $(0,0,0, .06, .24, .84, .87, .91)$, \\
& $(0,0,0,22,95,365,376,391))$ & $(0,0,0, .06, .24, .93, .96,1))$ \\
\hline & $((0,0,0,0,84,117,135,153)$, & $((0,0,0,0, .15, .21, .24, .27)$, \\
& $(0,0,0,0,84,171,189,216)$, & $(0,0,0,0, .15,3, .33, .38)$, \\
A-C- & $(0,0,0,0,84,234,252,270)$, & $(0,0,0,0, .15, .41, .44, .48)$, \\
D-E & $(0,0,0,0,84,288,306,333)$, & $(0,0,0,0,15, .51, .54, .59)$, \\
& $(0,0,0,0,84,351,369,390)$, & $(0,0,0,0, .15, .62, .65, .69)$, \\
& $(0,0,0,0,84,408,426,450)$, & $(0,0,0,0,15, .72, .75, .79)$, \\
& $(0,0,0,0,84,468,486,507)$, & $(0,0,0,0, .15, .83, .86, .89)$, \\
& $(0,0,0,0,84,525,543,567))$ & $(0,0,0,0, .15, .93, .96,1))$ \\
\hline & $((0,0,0,0,65,84,95,106)$, & $((0,0,0,0, .18, .23, .26, .29)$, \\
& $(0,0,0,0,65,117,128,145)$, & $(0,0,0,0, .18, .32, .35, .4)$, \\
& $(0,0,0,0,65,156,167,178)$, & $(0,0,0,0, .18, .43, .46, .49)$, \\
& $(0,0,0,0,65,189,200,217)$, & $(0,0,0,0, .18, .52, .55, .6)$, \\
& $(0,0,0,0,65,228,239,252)$, & $(0,0,0,0, .18, .63, .66, .7)$, \\
& $(0,0,0,0,65,263,274,289)$, & $(0,0,0,0, .18, .73, .76, .8)$, \\
& $(0,0,0,0,65,300,311,324)$, & $(0,0,0,0, .18, .83, .86, .9)$, \\
& $(0,0,0,0,65,335,346,361))$ & $(0,0,0,0, .18, .93, .96,1))$ \\
\hline
\end{tabular}

By using (15), we get

For $\alpha=1$,

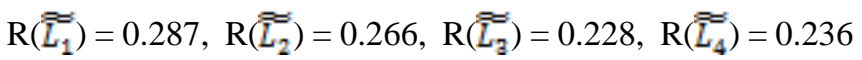

For $\alpha=0.5$,

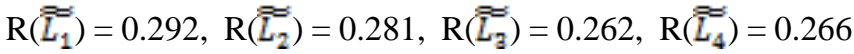

For $\alpha=0$,

$\mathrm{R}\left(\widetilde{L}_{1}\right)=\mathrm{R}\left(\widetilde{L}_{2}\right)=\mathrm{R}\left(\widetilde{L}_{2}\right)=\mathrm{R}\left(\widetilde{L}_{4}^{5}\right)=$

0.296

Here minimum ranks are $0.228,0.262$ and 0.296 for $\alpha=1$, $\alpha=0.5$ and $\alpha=0$ respectively.

So the critical path is $\mathrm{A}-\mathrm{C}-\mathrm{D}-\mathrm{E}$.

\section{RESULT AND DISCUSSION}

TABLE VII : Comparison Between Three Generalized Type-2 Fuzzy Numbers

\begin{tabular}{|c|c|c|c|c|c|c|c|c|c|}
\hline \multirow{2}{*}{ Path } & \multicolumn{3}{|c|}{$\begin{array}{c}\text { Generalized Type - } 2 \text { Trapezoidal } \\
\text { Fuzzy Number }\end{array}$} & \multicolumn{3}{|c|}{$\begin{array}{c}\text { Generalized Type - } 2 \text { Hexagonal } \\
\text { Fuzzy Number }\end{array}$} & \multicolumn{3}{|c|}{$\begin{array}{c}\text { Generalized Type - } 2 \text { Octagona } \\
\text { Fuzzy Number }\end{array}$} \\
\hline & $\alpha=0$ & $\alpha=0.5$ & $\alpha=1$ & $\alpha=\mathbf{0}$ & $\alpha=0.5$ & $\alpha=1$ & $\alpha=0$ & $\alpha=0.5$ & $\alpha=1$ \\
\hline $\mathrm{A}-\mathrm{B}-\mathrm{E}$ & 0.389 & 0.305 & 0.222 & 0.278 & 0.273 & 0.268 & 0.296 & 0.292 & 0.287 \\
\hline$A-C-E$ & 0.389 & 0.290 & 0.192 & 0.278 & 0.261 & 0.245 & 0.296 & 0.281 & 0.266 \\
\hline$A-C-D-E$ & 0.389 & 0.256 & 0.129 & 0.278 & 0.238 & 0.198 & 0.296 & 0.262 & 0.228 \\
\hline $\mathrm{A}-\mathrm{D}-\mathrm{E}$ & 0.389 & 0.265 & 0.142 & 0.278 & 0.243 & 0.208 & 0.296 & 0.266 & 0.236 \\
\hline
\end{tabular}

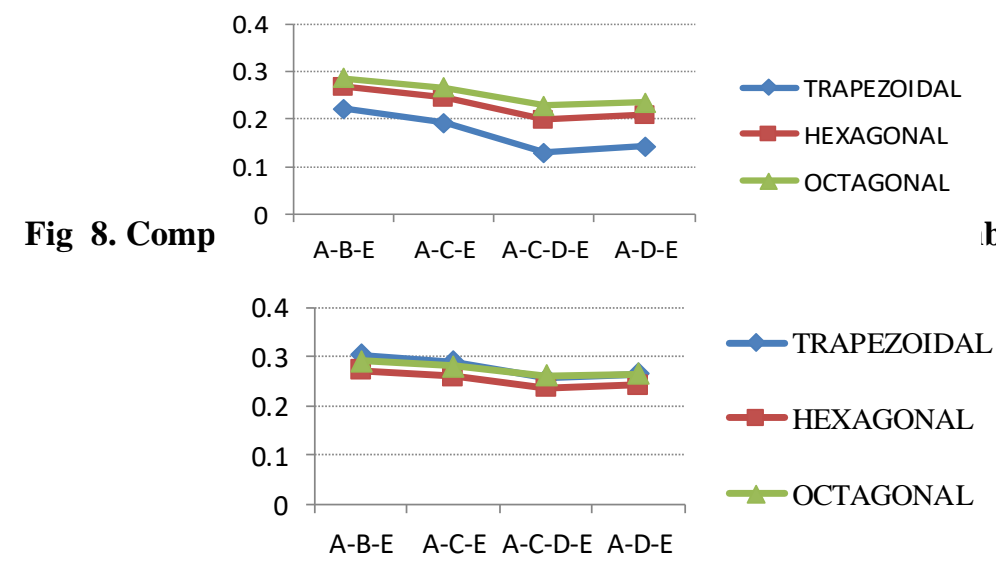

Fig 9. Comparison among three Type-2 Generalized Fuzzy Numbers if $\alpha=0.5$ 


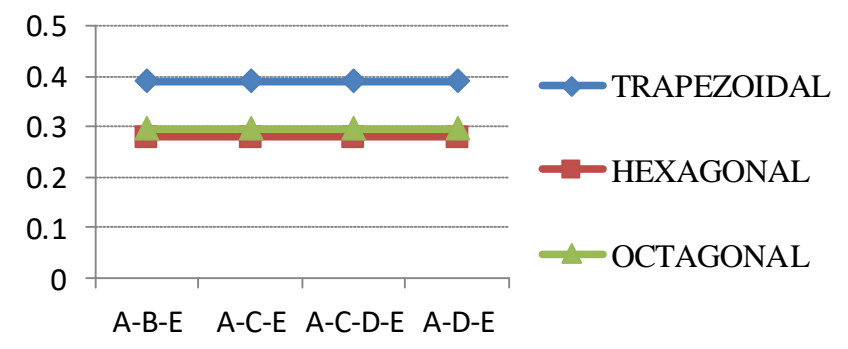

Fig 10. Comparison among three Type-2 Generalized Fuzzy Numbers if $\boldsymbol{\alpha}=0$

\section{CONCLUSION}

Critical path method (CPM) has been widely used in managing complicated project network models in real world applications. This paper has proposed an algorithm for finding critical path based on various type-2 fuzzy quantities using proposed fuzzy ranking functions for ordering type-2 fuzzy arc lengths. Moreover, the analysis has been made among the generalized type-2 trapezoidal, hexagonal and octagonal fuzzy numbers in order determine the effective ranking value for finding the critical path. Finally, the results show that Type-2 trapezoidal fuzzy number is more effective to determine the activity criticalities to find the critical path based on the proposed ranking index

\section{REFERENCES}

1. V. Sireesha and N. Ravi Shankar, "A new approach to find total float time and critical path in a fuzzy project network", International Journal of Engineering Science and Technology, Vol. 2, 2010, pp. 600 - 609.

2. Shakeela Sathish and K. Ganesan, "A simple approach to fuzzy critical path analysis in Project networks", International Journal of Scientific \& Engineering Research, Vol. 2, 2011, pp. 1 - 6.

3. N. Ravi Shankar, B. Pardhasaradhiand S. Suresh Babu, "Fuzzy Critical Path Method Based on a new approach of Ranking Fuzzy Numbers using Centroid of Centroids", International Journal of Fuzzy System Applications, Vol. 3, 2013, pp. 16 - 31.

4. S. Elizabeth and L. Sujatha, "Fuzzy Critical Path Problem for Project Network", International Journal of Pure and Applied Mathematics, Vol. 85 , 2013, pp. 223 - 240.

5. P. Jayagowri and G. Geetharamani, “A Critical Path Problem Using Intuitionistic Trapezoidal Fuzzy Number”, Applied Mathematica Sciences, Vol. 8, 2014, pp. 2555 - 2562.

6. MH. Oladeinde and CA. Oladeinde, "Metric Distance Ranking Technique for Fuzzy Critical Path Analysis", J. Appl. Sci. Environ. Manage,Vol. 18, 2014, pp. 583 -589.

7.D. Stephen Dinagar and D. Abirami, "A note on fuzzy critical path analysis in project networks", International Journal of Current Research, Vol.6, 2014, pp.7458 - 7465.

8. D. Stephen Dinagar and D. Abirami, "A Study on Critical Path Method Using Interval Valued Fuzzy Numbers", International Journal of Mathematics and its applications, Vol. 4, 2016, pp. 293 - 301.

9. D. Stephen Dinagar and D. Abirami, "A Distinct Method to find the Critical Path and Total Float under Fuzzy Environment”, International Journal of Scientific Research Engineering \& Technology , Vol. 6, 2017, pp. 90 - 94.

10. S. Vimala and S. Krishna Prabha, "Solving Fuzzy Critical Path Problem Using Method of Magnitude", International Journal of Scientific \& Engineering Research, Vol. 6, 2015, pp. 1362 - 1370.

11.S. Narayanamoorthy and S. Maheswari, "The Intelligence of Octagonal Fuzzy Number to determine the Fuzzy Critical Path: A New Ranking Method", Hindawi Publishing Corporation Scientific Programming, Volume 2016, 2016, pp. 1 - 8.

12. V. Pusparaj and P. John Paul, "A Study on Fuzzy Critical Path Method Based On Metric Distance Ranking Of Fuzzy Numbers with Conventional Method", International Journal of Mathematics Trends and Technology, Vol. 40, 2016, pp. 230 - 236.
13. A. Hari Ganesh and A. Helen Shobana, "On Fuzzy Shortest Path Algorithm based on Type-2 Generalized Hexagonal Fuzzy Number", Turkish Journal of Fuzzy Systems, Vol. 7, 2016, pp. 1-12 14. V. Anusuya and P. Balasowandari, "Critical Path with Various Distances of Type-2 Fuzzy Numbers", International Journal of Fuzzy Mathematical Archive, Vol. 13, 2017, pp. 1 - 7.

15. Jing Wang et. al., "Multi-Criteria Decision-Making Method Based on Type - 2 Fuzzy Sets", Filomat, Vol. 2 , 2017, pp.431 - 450.

16.L.A. Zadeh, “Fuzzy Sets”, Inform. Control, Vol.8, 1965, pp. 338 353.

17. M. Clement Joe Anand and M. Lathamaheswari, “A New eneralized Normal Type-2 Trapezoidal Fuzzy Number”, Global Journal of Pure and Applied Mathematics, Volume 12, 2016, pp. 565 - 574.

\section{AUTHORS PROFILE}

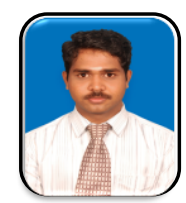

Dr. A. Hari Ganesh received his Master degree, Master of Philosophy and Doctor of Philosophy in Mathematic from Bharathidasan University, Trichy, Tamil Nadu, He has qualified Tamil Nadu State Eligibility Test (TNSET) in the year 2016. He is currently an Assistant Professor in the department of Mathematics at Poombuhar College(Autonomous), Nagapattinam, Tamil Nadu. He has published around 20 papers in National and International Peer Review Journals. His areas of interest are Fuzzy Mathematics and Number Theory.

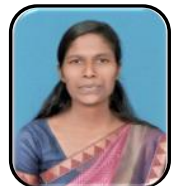

A. Helen Shobana received her Master Degree and Master of Philosophy from Bharathidasan University, Trichy, Tamil Nadu. She has qualified Tamil Nadu State Eligibility Test (TNSET) for Lectureship in the year 2017. Now she is a Ph.D candidate in Bharathidasan University,

Mathematics. Trichy, Tamil Nadu. Her area of interest is Fuzzy 